\title{
A 19-isolate reference-quality global pangenome for the fungal wheat pathogen Zymoseptoria tritici
}

\author{
Thomas Badet ${ }^{1}$, Ursula Oggenfuss ${ }^{1}$, Leen Abraham¹', Bruce A. McDonald ${ }^{2}$ and Daniel Croll ${ }^{1 *}$ (D)
}

\begin{abstract}
Background: The gene content of a species largely governs its ecological interactions and adaptive potential. A species is therefore defined by both core genes shared between all individuals and accessory genes segregating presence-absence variation. There is growing evidence that eukaryotes, similar to bacteria, show intra-specific variability in gene content. However, it remains largely unknown how functionally relevant such a pangenome structure is for eukaryotes and what mechanisms underlie the emergence of highly polymorphic genome structures.

Results: Here, we establish a reference-quality pangenome of a fungal pathogen of wheat based on 19 complete genomes from isolates sampled across six continents. Zymoseptoria tritici causes substantial worldwide losses to wheat production due to rapidly evolved tolerance to fungicides and evasion of host resistance. We performed transcriptomeassisted annotations of each genome to construct a global pangenome. Major chromosomal rearrangements are segregating within the species and underlie extensive gene presence-absence variation. Conserved orthogroups account for only $\sim 60 \%$ of the species pangenome. Investigating gene functions, we find that the accessory genome is enriched for pathogenesis-related functions and encodes genes involved in metabolite production, host tissue degradation and manipulation of the immune system. De novo transposon annotation of the 19 complete genomes shows that the highly diverse chromosomal structure is tightly associated with transposable element content. Furthermore, transposable element expansions likely underlie recent genome expansions within the species.
\end{abstract}

Conclusions: Taken together, our work establishes a highly complex eukaryotic pangenome providing an unprecedented toolbox to study how pangenome structure impacts crop-pathogen interactions.

\section{Background}

Microbial species harbour substantial functional diversity at the level of gene presence-absence variation [1]. Genes not fixed within a species (i.e. accessory genes) can account for a large fraction of the full gene repertoire (i.e. the pangenome). In bacteria, the proportion of core genes in the pangenome can range from 5 to $98 \%$ and challenge taxonomic classifications $[2,3]$. The wide spectrum of pangenome sizes across species can be associated with the species distribution and lifestyle [4]. Species showing a wide geographical distribution and large population sizes characterized by frequent genetic exchange tend to have expansive, open pangenomes [5].

\footnotetext{
* Correspondence: daniel.croll@unine.ch

'Laboratory of Evolutionary Genetics, Institute of Biology, University of Neuchâtel, Neuchâtel, Switzerland

Full list of author information is available at the end of the article
}

In microbial pathogens, accessory genes play a major role in virulence and environmental adaptation [6-8]. The notion of a pangenome led to the discovery that major elements of intra-specific variation are often ignored in studies relying on a single reference genome. Large pangenomes also can challenge association studies aiming to identify the genetic basis of phenotypic traits because mapping is often performed against a single reference genome, making potentially relevant genetic variation inaccessible $[9,10]$. Despite their importance for unraveling the genetic basis of adaptive evolution, only a very limited number of eukaryotic species have well established pangenomes.

Copy number variation including gene deletion generates intraspecific gene content variation in nearly all species [11]. This variation can create extreme variance in fitness and promote adaptive evolution [12-15]. In 
plant pathogens, the ability to infect a host often relies on the secretion of effector proteins that interfere with the host cell machinery [16-18]. Host plants evolved cognate resistance proteins that are able to recognize effector proteins and trigger immunity [19]. Gains and losses of effector genes can therefore have a major impact on the outcome of host-pathogen interactions and challenge food security. Recent studies on fungal pathogens highlighted that genes showing presence-absence variation are enriched for predicted effectors [14, 20, 21]. Effectors and transposable elements (TEs) are often tightly associated with fast-evolving compartments of the genome [22, 23], also known as the "two-speed" genome architecture [24]. However, how TEs impact the birth and death of effectors in fast-evolving compartments remains largely unclear [6, 25]. The construction of pathogen pangenomes enabled crucial insights into functional diversity and the evolutionary trajectories of host adaptation. Recent pangenome analyses of four fungal species including opportunistic pathogens revealed that between $\sim 9$ and $19 \%$ of the pangenome is accessory. Accessory gene localization was preferentially in subtelomeric regions, suggesting both a mechanistic link to repeat-rich regions and relaxation of selective constraints [26]. The wheat pathogen Zymoseptoria tritici was found to have one of the largest eukaryotic pangenomes with an estimate of at least $42 \%$ of all genes being accessory [27]. However, eukaryotic pangenomes remain shallow and are often based on not fully resolved chromosomal sequences.

Fungal plant pathogens such as $Z$. tritici show extreme cases of genome plasticity. The reference genome of $Z$. tritici has 21 chromosomes, of which eight are accessory and segregate presence-absence variation in populations [28]. The pathogen rapidly evolved virulence on resistant wheat cultivars and has overcome all current fungicides [29-31]. Host adaptation was driven among other factors by the rapid deletion of an effector gene and structural rearrangements [32-34]. Pathogen populations are highly diverse with high rates of recombination [35-37]. Meiosis can trigger large chromosomal rearrangements and lead to aneuploid chromosomes in the species $[38,39]$. A pangenome constructed for five Z tritici isolates revealed that chromosome length variation segregating within populations was mainly due to the presence-absence variation of large TE clusters $[27,40]$. Furthermore, accessory genes tended to form clusters dispersed along chromosomes. Accessory genes also tended to be in closer proximity to TEs than core genes and were therefore more likely to be affected by epigenetic silencing [27]. However, the constructed pangenome was very likely incomplete given the fact that four of the genomes originated from isolates collected in the same year from two nearby fields. Furthermore, accessory genes were enriched for pathogenesis-related functions but the pangenome size did not reach saturation. Given the global impact of the pathogen and the importance of accessory genes for adaptive evolution, a comprehensive pangenome capturing worldwide genetic diversity is essential.

In this study, we construct the pangenome of $Z$. tritici by including 19 isolates sampled from six different continents and covering the global distribution of the pathogen. We test to what extent the species segregates chromosomal rearrangements and how this impacts gene presence-absence variation at loci relevant for pathogenicity. We also analyse whether TE content is polymorphic within the species and may contribute to genome size evolution.

\section{Results}

\section{Major chromosomal rearrangements segregating within the species}

We constructed a global pangenome of $Z$. tritici based on 19 isolates sampled from six continents and 13 different countries (Fig. 1a, b). The isolates included the previously described reference isolate IPO323 sampled in the Netherlands and four isolates that were isolated from two nearby fields in Switzerland [27, 28, 40]. The geographic regions of origin of the 19 isolates recapitulate a significant environmental gradient in mean annual temperature and humidity and span the distribution range of the species. The sampling period ranges from 1984 (IPO323) to 2010 (CRI10). Fungicide applications against $Z$. tritici became widespread in the 1990s and early 2000s; hence, the sampling covers both pre- and post-fungicide treatment regimes. We sequenced longread PacBio SMRTbell libraries to a depth of 40-110X and $\sim 20 \mathrm{~kb}$ read coverage in order to generate chromosomelevel assemblies. Assembly sizes ranged from $37.13 \mathrm{Mb}$ (IR01_48b) to $41.76 \mathrm{Mb}$ (Aus01) (Fig. 1c).

We recovered all eight known accessory chromosomes of the species but no additional chromosome. The accessory chromosome 18 is most often missing. Together, the 8 accessory chromosomes display an average size variation of $\sim 37 \%$ across all isolates and a maximum of $60 \%$ for chromosome 14 (Fig. 2a). For core chromosomes, the average size variation accounts for $16 \%$ of chromosome length going up to $23 \%$ for chromosome 7 . We identified a major deletion spanning $406 \mathrm{~kb}$ and encompassing 107 genes on the right arm of core chromosome 7 of the Yemeni isolate (YEQ92; Fig. 2b lower panel). The same isolate had chromosome 15 fused to the right arm of chromosome 16. The fusion event is supported by aligned PacBio reads spanning the region between the two chromosomal segments (Additional file 1: Figure S1). The resulting chromosome is $1.20 \mathrm{Mb}$ long and $49.5 \mathrm{~kb}$ shorter than the sum of the homologous chromosomes 15 and 16 of the IPO323 reference genome. Approximately $90 \%$ of the genes on the IPO323 chromosome 15 and 16 belong to accessory 


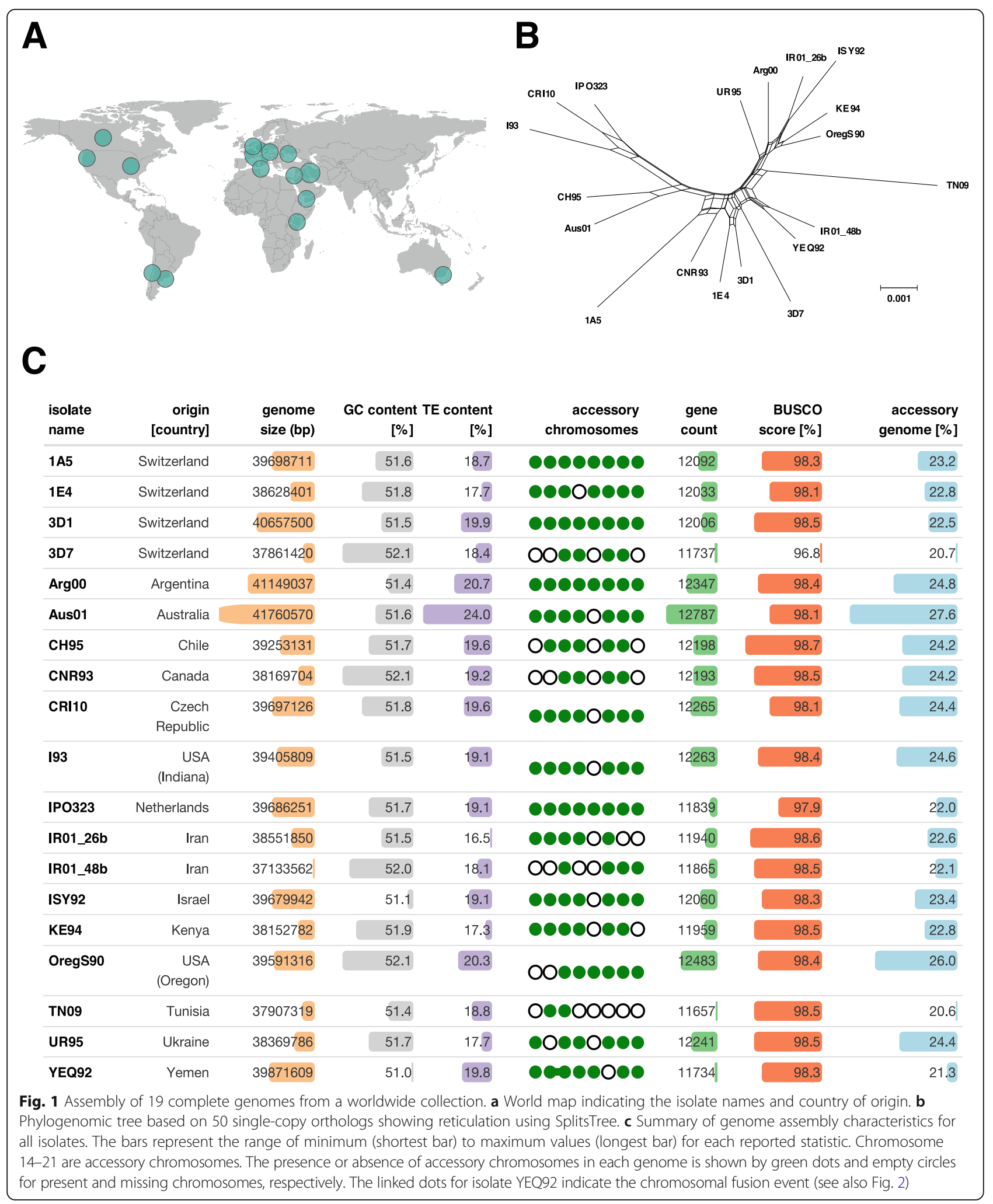

orthogroups, as they lack an ortholog in at least one of the other isolates. We find that the chromosomal fusion deleted about $150 \mathrm{~kb}$ affecting 1 and 12 genes on chromosomes 15 and 16, respectively (Fig. 2b upper panel). We further assessed genome completeness using BUSCO analyses. All genomes exceed the completeness of the fully finished IPO323 reference genome (97.9\%) with the exception of isolate 3D7 (96.8\%; Fig. 1c). 


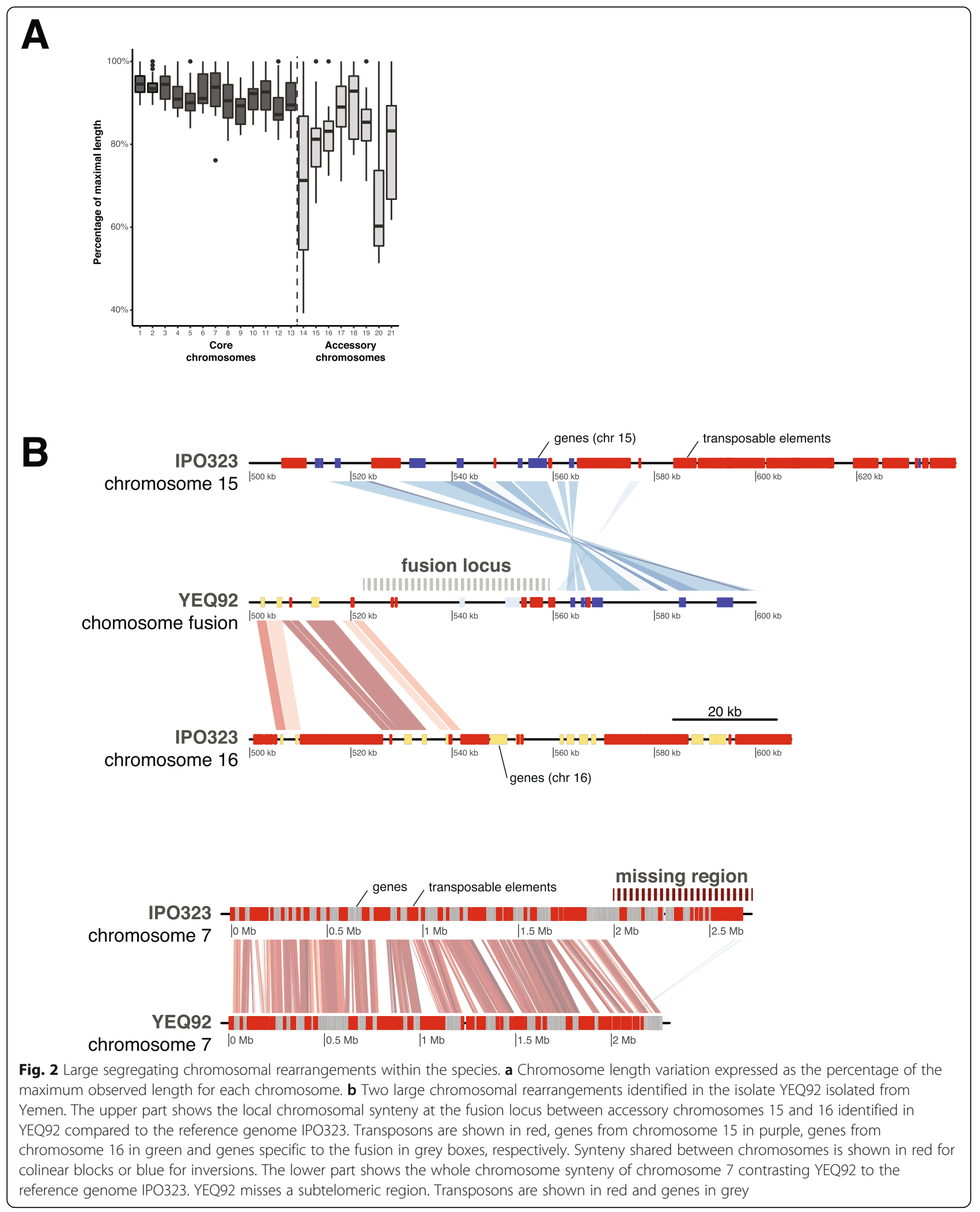


Substantial gene content variation across the pangenome We generated RNAseq data to identify high-confidence gene models in all 14 newly assembled genomes based on a splice-site informed gene prediction pipeline. The total gene count varied between 11,657 and 12,787 gene models (Fig. 1c). We assigned all genes to orthogroups using protein homology and constructed a pangenome of all 19 complete genomes. The pangenome consists of a total of 229,699 genes assigned to 15,474 orthogroups. The number of genes assigned per orthogroup varies among isolates (Fig. 2b). Approximately $99.8 \%$ of all orthogroups $(15,451)$ are single-gene orthogroups and $60 \%$ of all orthogroups are shared among all 19 isolates (9193 core orthogroups). Around 96\% of the core orthogroups (8829 out of 9193) have conserved gene copy numbers among isolates. Furthermore, we find that $30 \%$ of all orthogroups are shared between some but not all genomes (4690 accessory orthogroups) and 10\% of the orthogroups are composed of genes found in a single genome only (1592 singletons; Fig. 3a, b; Additional file 2: Table S1).

To infect wheat, Z. tritici relies on specific gene functions $[41,42]$. Effectors play a major role in establishing infection and exploiting host resources. Hence, we analysed how gene functions were structured across the pangenome components. Core orthogroups showing variation in gene-copy number among isolates include five encoding predicted effectors. Both accessory proteins and overall effector proteins are less conserved than core proteins at the amino acid level (Additional file 1: Figure S2). A total of 3.5\% (691) of all orthogroups encode at least one predicted effector. Among orthogroups encoding at least one predicted effector, 31\% were conserved among all isolates (219), 63\% were accessory (436), and 5\% were found in only one isolate (36 singletons). Notably, 99\% of the predicted effector genes are located on core chromosomes. In addition to effectors, enzymes enabling access to nutrients are important pathogenicity components. We identified a total of 4742 annotated carbohydrate-degrading enzymes (CAZymes) clustered into 263 orthogroups. Notably, 92\% of the orthogroups encoding CAZymes were conserved among all isolates (Fig. 3a). CAZymes grouped into 123 subfamilies. Glycoside hydrolases $(\mathrm{GH})$ are the largest family and account for $57 \%$ of all annotated CAZymes (151 orthogroups for 2717 genes). Glycosyl transferases (GT) are the second most abundant family with 1188 genes and 66 orthogroups $(25 \%$ of all CAZymes) (Fig. 3c). We also identified 33 orthogroups encoding for auxiliary activities (AA), 9 for carbohydrate esterase activity (CE), 6 for carbohydrate-binding modules (CBM) and 3 for polysaccharide lyase activity (PL). The PL family includes $29 \%$ accessory genes. Across CAZyme families, $0-10 \%$ of the genes are accessory (Fig. 3c). We found a singleton GH43 subfamily gene in the genome of the Australian isolate (Aus01).

The production of secondary metabolites contributes significantly to virulence and competitive abilities of fungal pathogens. We identified between 29 and 33 secondary metabolite gene clusters per genome depending on the isolate. A total of $70 \%$ of all genes predicted as components of a biosynthetic gene cluster are conserved between all isolates and 30\% are accessory (Fig. 3d, Additional file 1: Figure S3). Of the 147 orthogroups annotated as encoding biosynthetic or biosyntheticadditional proteins in the pangenome, 87, 92, 111 and 112 have a homologue with $>50 \%$ identity in the four closely related sister species $Z$. passerinii, $Z$. ardabiliae, $Z$. pseudotritici and $Z$. brevis, respectively (Additional file 1 : Figure S4). We identified 39 syntenic gene clusters in the pangenome classified into 12 type 1-polyketide synthase (PKS), 11 non-ribosomal peptide synthetase (NRPS), four terpene, one type 3-PKS, one siderophore, one indole and eight unclassified clusters. Sixteen $(40 \%)$ of the identified syntenic clusters show presence-absence variation. In the $\mathrm{CH} 95$ isolate, a gene cluster on chromosome 7 was annotated as unclassified but annotated as a NRPS in 17 other isolates and absent from the IPO323 reference genome. The sole indole and type 1-PKS clusters located on chromosomes 5 and 10 , respectively, were only found in isolate TN09. Two type 1-PKS and one NRPS cluster were missing in the isolates YEQ95, Aus01 and IPO323, respectively. Among the 39 identified syntenic gene clusters, 23 included a predicted effector and nine included a gene annotated as a cell-wall degrading enzyme.

The emergence of fungicide tolerance in $Z$. tritici is a major threat to wheat production. Succinate dehydrogenase $(\mathrm{SDH})$ inhibitors are commonly used as control agents [31, 43]. We identified five SDH orthologs, of which three were conserved among all genomes (SDHB, SDHC and SDHD subunits). We find two distinct SDHC paralogs SDHC2 and SDHC3 in 11 and two isolates, respectively. The SDHC3 paralog conferring standing resistance to SDH inhibitors is located adjacent to a large cluster of TEs, suggesting that chromosomal rearrangements were underlying the paralog emergence (Fig. 3e). Genes encoding major facilitator superfamily (MFS) transporters, which can confer multidrug resistance in $Z$. tritici [44], grouped into 336 orthogroups for a total of 5787 genes (Additional file 2: Table S2). We find that $39(11 \%)$ of these orthogroups are part of a predicted secondary metabolite gene cluster and one is an annotated CAZyme from the GH78 family. Overall, the results reveal that gene families essential for pathogenicity and fungicide resistance show unexpectedly high levels of presence-absence variation in the $Z$. tritici pangenome. 
A

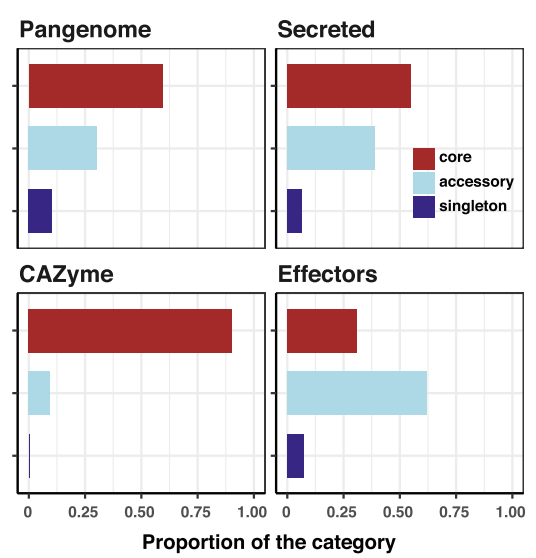

C

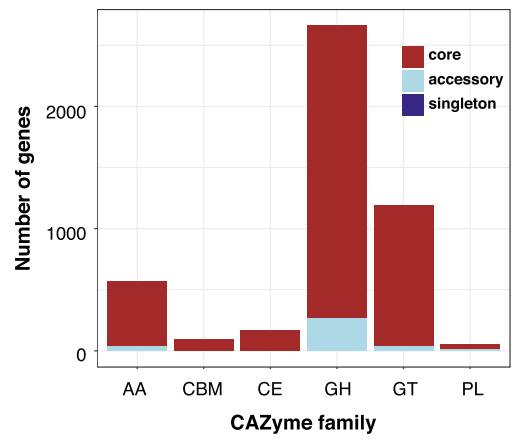

B
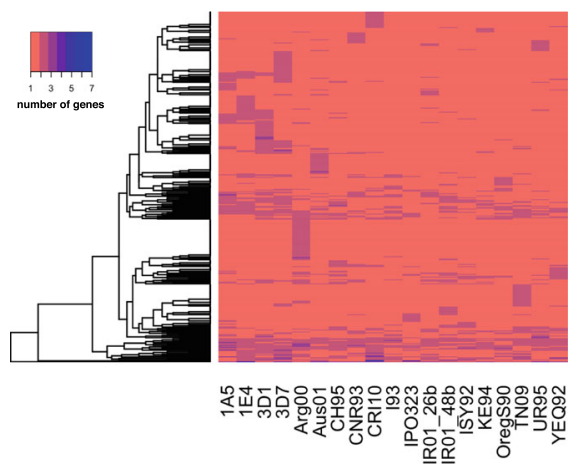

D

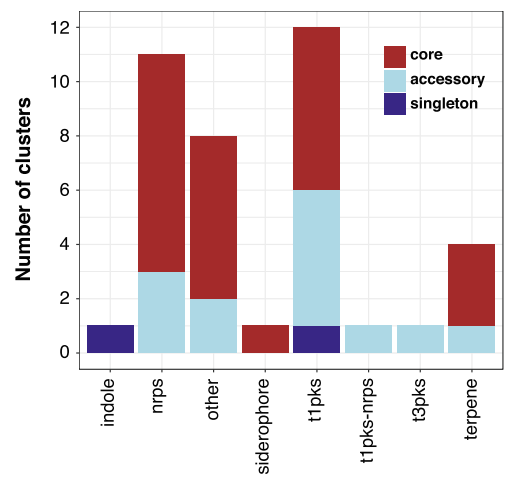

E

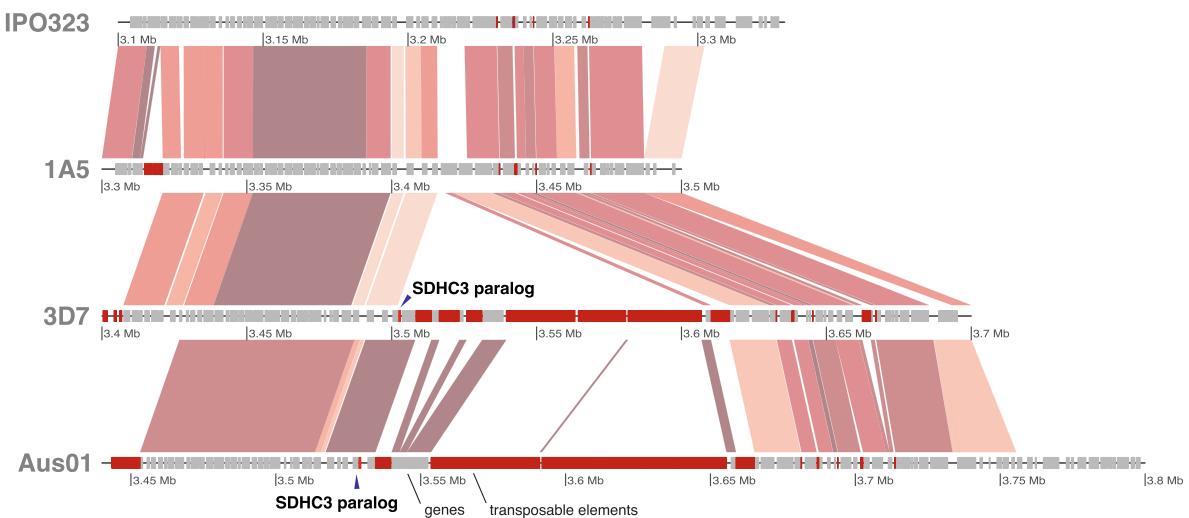

Fig. 3 Construction and analysis of the Zymoseptoria tritici pangenome. a Proportions of core orthogroups (present in all isolates), accessory orthogroups (present $\geq 2$ isolates but not all) and singletons (present in one isolate only) across the pangenome (upper-left). The proportions of core, accessory and singleton categories are shown for orthogroups coding for secreted proteins (upper-right), carbohydrate-active enzymes (CAZymes; lower-left) and effectors (lower-right). b Gene copy number variation in core orthogroups across the 19 genomes. c Pangenome gene count across six CAZyme families. Families are divided into glycoside hydrolase (GH), glycosyl transferase (GT), auxiliary activity (AA), carbohydrate esterase (CE), carbohydrate-binding modules (CBM) and polysaccharide lyase activity (PL) categories. $\mathbf{d}$ Pangenome categories of secondary metabolite gene clusters. e Synteny plot of succinate dehydrogenase (SDH) paralogs mediating fungicide resistance. The SDHC3 locus on chromosome 3 is shown for isolates 3D7 and Aus01 both carrying the paralog. IPO323 and 1A5 lack SDHC3. The position of the SDHC3 paralog is shown using dark arrows. Genes are coloured in grey and transposable elements in red 
Strong expression variation across major gene functions Differential gene expression is a major driver of intraspecific phenotypic differences. We performed mRNAsequencing of all 19 isolates grown on minimal media. Minimal media induces filamentous growth of $Z$. tritici, mimicking the morphology and nutrient starvation that occurs early during plant infection. We investigated isolate-specific gene expression by self-mapping RNAseq reads to each isolate's genome assembly. Overall, 91.3\% of the genes show expression on minimal media and $68 \%$ have expression of more than 10 counts per million (CPM) (Fig. 4a). Core genes have higher expression than accessory genes (Additional file 1: Figure S5). Among the genes showing no expression on minimal media, 501 are predicted effector genes ( $8 \%$ of predicted effectors), 93 are predicted CAZymes (2\% of CAZymes) and 838 are members of a predicted gene cluster $(10 \%$ of all gene cluster genes). CAZymes are overall highly expressed on minimal media $(\sim 77 \%$ with $\mathrm{CPM}>10)$ when compared to effectors $(\sim 45 \%$ with $\mathrm{CPM}>10)$ and gene cluster genes $(\sim 60 \%$ with $\mathrm{CPM}>10)$ (Fig. $4 \mathrm{a})$. About $53 \%$ of core single-copy orthogroups with non-zero expression have a coefficient of variation $>50 \%$ (Fig. 4b). Similarly, $\sim 68 \%$ of CAZymes and $\sim 60 \%$ of genes that are part of a secondary metabolite cluster have expression coefficient of variation $>50 \%$. In contrast, about $90 \%$ of orthogroups encoding predicted effectors have a coefficient of variation $>50 \%$, together with $\sim 81 \%$ of accessory orthogroups.

To identify broad patterns in the pangenome expression landscape, we performed a clustering analysis of all core single-gene orthogroups. We find that expression clustering does not reflect the geographical origin or genetic distance with the exception of the four Swiss isolates (1A5, 1E4, 3D1 and 3D7; Additional file 1: Figure S6). We also analysed the impact of copy-number variation on average expression and find that single-copy orthologs are on average more highly expressed. In addition, we show that gene expression rapidly decreases if an orthogroup includes 2-8 paralogs (Fig. 4c).

\section{A highly variable transposable element content within the species}

TEs are drivers of pathogen evolution by generating adaptive genetic variation. To identify genes with a potential role in the mobilization of TEs, we analysed large homology groups. Among the orthogroups with 10 or more paralogs, $\sim 88 \%$ of the genes encode proteins without homology in databases, $\sim 7 \%$ of the genes encode nucleic acid binding functions (GO:0003676), $\sim 2 \%$ of the genes encode a retrotransposon nucleocapsid (GO: $0000943)$ and $\sim 1.5 \%$ of the genes encode a DNA integration domain (GO:0015074). Orthogroups with 10 or more paralogs are all accessory. For isolates sharing the same large orthogroups, we identified variability in the gene copy number within those orthogroups. Indeed, the isolates Aus01 and OregS90 have 26 and 16 orthogroups, respectively, with more than 10 assigned genes. The isolates 193 and Arg00 count between one and six orthogroups and nine other isolates have no orthogroups larger than ten genes (Fig. 4d). Altogether, these results suggest that large orthogroups (>10 genes) essentially regroup genes that are encoded by TEs. Our data also indicates regional TE-driven genome expansions given the enlarged genome sizes in Australian and North American isolates.

To elucidate the role of transposition on generating genomic variation, we screened the 19 genomes for TE content. For this, we jointly analysed all complete genomes to exhaustively identify repetitive DNA sequences. We identified a total of 304 high-quality TE family consensus sequences grouped into $22 \mathrm{TE}$ superfamilies. The $\mathrm{GC}$ content of the consensus sequences is highly variable, ranging from 23 to 77\% (Additional file 1: Figure S7). On average, TE superfamilies have a GC content lower than $50 \%$, except for unclassified SINE families (RSX; GC\% 50.6). The genomic TE content ranges from $16.48 \%$ (IR01_26b) to $23.96 \%$ (Aus01) and is positively correlated with genome size (cor $=0.78, p<0.001$; Fig. $5 \mathrm{a}$ ). Genome size correlates with genome-wide TE proportions on both core and accessory chromosomes but is negatively correlated with the proportion of coding sequences (Additional file 1: Figure S8 and Figure S9). The average length of individual TEs ranges from 102 to 51, $298 \mathrm{bp}$ with the Helitron superfamily having the higher average length (Additional file 1: Figure S10S11). The largest element is an unclassified LTR (RLX_LARD_Thrym) on chromosome 7, the size of which ranges from $6282 \mathrm{bp}$ in CNR93 to 59,390 bp in ISY92. This particular LTR is present at the locus only in 18 isolates including ISY92, which has a fragmented secondary copy on chromosome 3. The RLX_ LARD_Thrym insertion on chromosome 7 overlaps with the ribosomal DNA locus and showed far above average mapped PacBio read coverage $(\sim 250 \times)$.

The genome-wide content of TEs shows substantial variation among the 19 isolates; however, the relative abundance of different TE superfamilies is relatively conserved with LTR Gypsy, unclassified TIR and LTR Copia elements being the most frequent (Fig. 5b). Accessory chromosomes contain consistently higher proportions of TEs compared to core chromosomes $(26-41 \%$ versus 17-24\%; Fig. 5c). Aus01 and OregS90 isolates showed the highest TE content. Interestingly, the Aus01 genome shows LINE I, LTR Gypsy and LTR Copia familyspecific expansion compared to other genomes. In contrast, the genome of OregS90 shows evidence for expansions of Helitron, LTR Gypsy and LTR Copia families. On average, $10 \%$ of all TEs overlap with genes. 


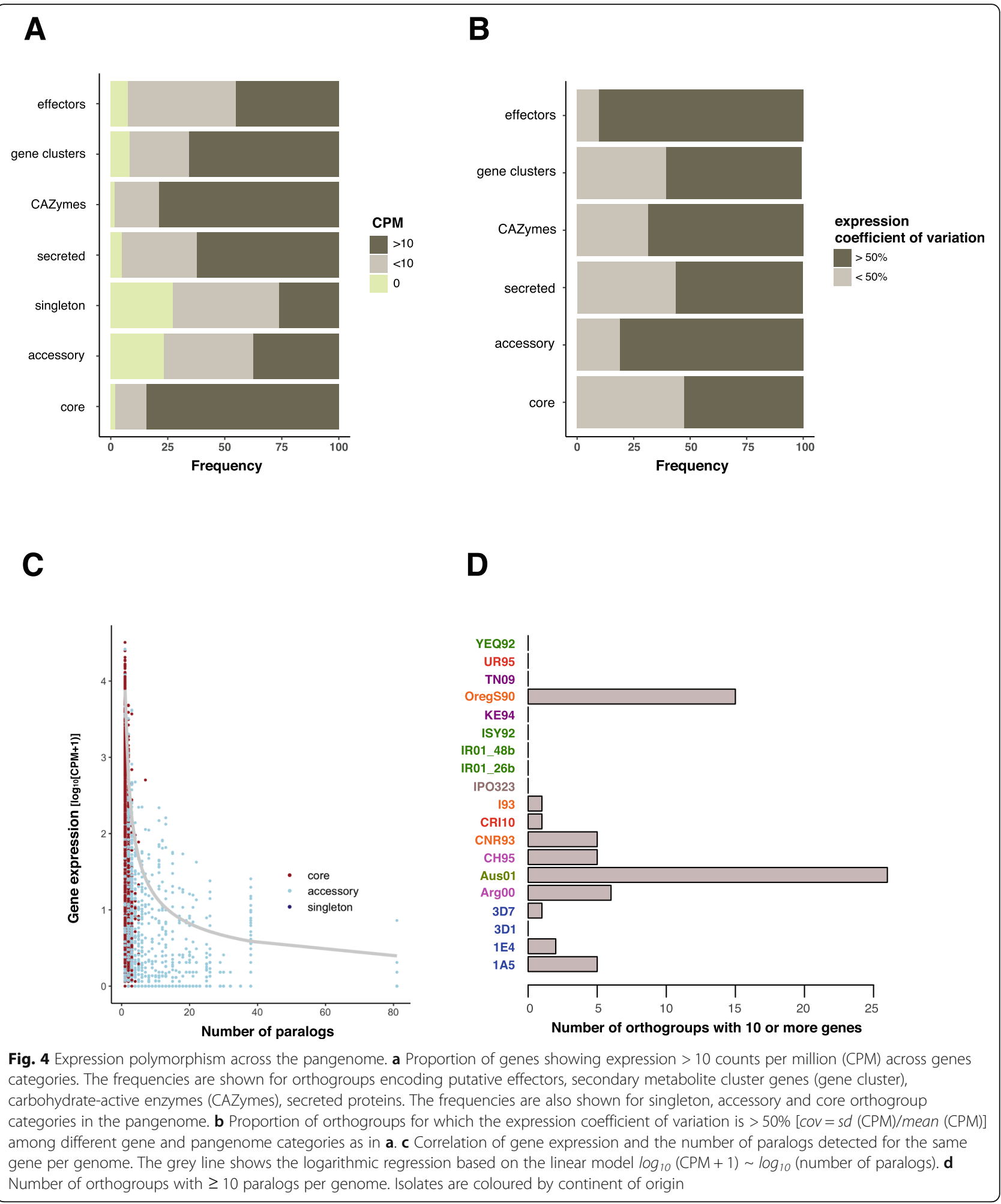

Overall, singleton and accessory genes tend to be closer to TEs and contain more often TE insertions than core genes (Additional file 1: Figure S12-S13). The isolates Aus01 and OregS90 have $12.8 \%$ and $12.4 \%$ of all TEs overlapping with genes, respectively. In addition, Aus01 and OregS90 isolates have $7.4 \%$ and $5.4 \%$ of all genes that overlap with TEs, respectively (Additional file 1: Figure S14). The composition of TEs inserted into genes reflects the overall TE composition in the genome, with more abundant TEs being more often inserted into 


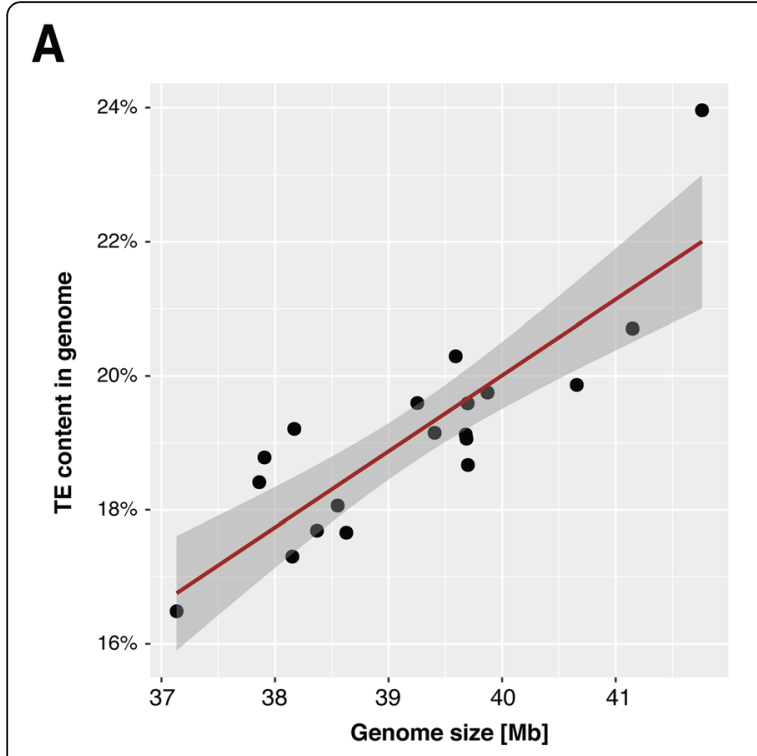

\section{B}

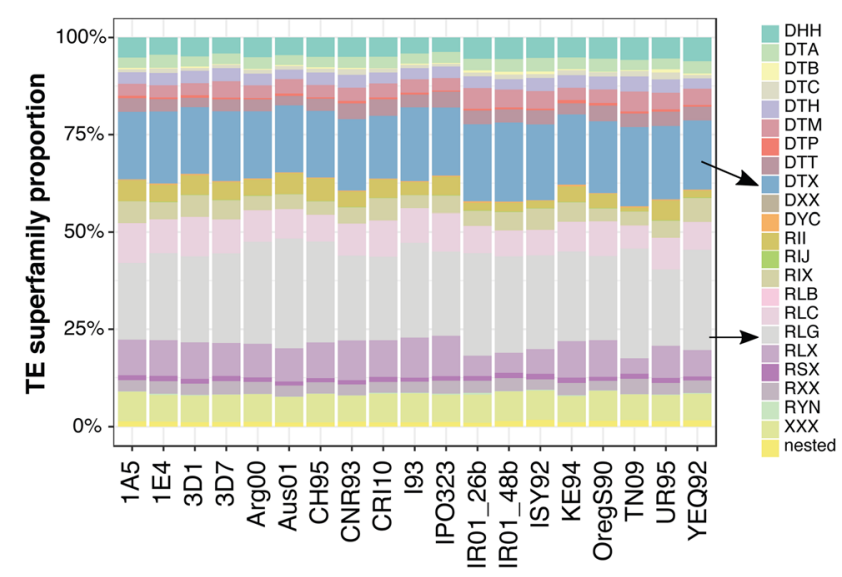

\section{C}

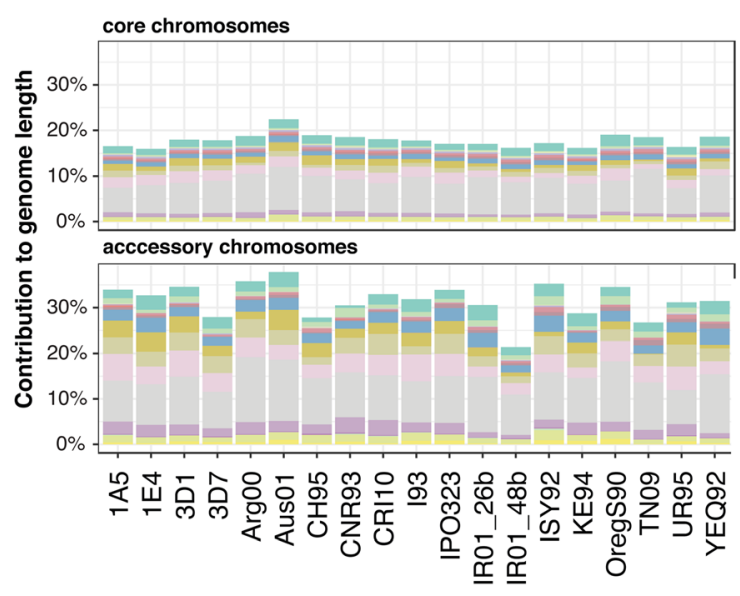

D
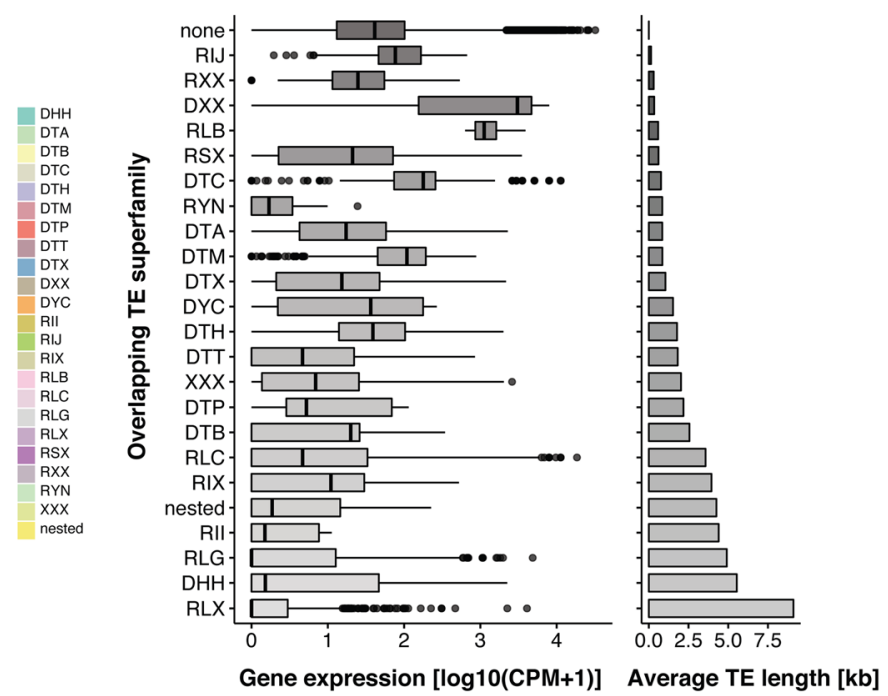

Fig. 5 Transposable elements (TEs) and genome size variation. a Contribution of TEs (\%) to total genome size across the 19 isolates. b Relative frequency of the 23 TE superfamilies across all genomes with 100\% referring to the total TE content of the respective genome. c Contribution of TE superfamilies to core and accessory genome size across the 19 isolates. $\mathbf{d}$ Expression of genes affected by TE insertions (grouped by TE superfamilies; left panel) and the mean TE length in the genome (grouped by TE superfamilies; right panel)

genes (Additional file 1: Figure S15). TEs can carry their own regulatory sequences and are often epigenetically silenced by the host. We found that orthogroups comprising a gene within $100 \mathrm{bp}$ distance of a TE show stronger expression variation $(\sim 62 \%$ of orthogroups with a coefficient of variation $>50 \%)$ compared to other orthogroups ( $~ 54 \%$ of orthogroups with a coefficient of variation > $50 \%)$ (Additional file 1: Figure S16-S17). We also found that different TE superfamilies have contrasting effects on gene expression, with longer TEs having more drastic effects (Fig. 5d). On average, genes with an inserted TE have lower expression levels (log10 CPM $\sim 1.7$-fold) and a higher coefficient of variation ( $\log 10 \mathrm{CPM} \sim 2$-fold) compared to genes without an inserted TE (Additional file 1: Figure S18).

\section{TE transcription correlates with relative frequency across isolates}

Class I TEs replicate through an RNA intermediate and class II through a DNA intermediate. Nevertheless, class 
II TEs can also transcribe into RNA. To gain insights into the mechanisms of proliferation, we analysed the relative abundance of TE-derived transcripts across all genomes. The highly repetitive nature of TEs typically prevents expression quantification at the individual copy level. Hence, we focused on normalized TE expression across all copies. Overall, more than $70 \%$ of the TE families have non-zero transcription levels. This is consistent with recent findings of pervasive transcription of TEs in the $Z$. tritici genome under nutrient stress and during infection [45]. We find that the largest TE family, an unclassified LTR identified as RLX_LARD_Thrym, was the most transcribed with an average $\log _{10} \mathrm{CPM} \sim$ 4.2 (Fig. 6a). An unclassified DTX-MITE is the second most transcribed TE with an average $\log _{10} \mathrm{CPM} \sim 3.6$ followed by an unclassified TE (XXX_Hermione with an average $\log _{10}$ CPM $~ 3.4$ ). At the superfamily level, LINEs have the highest expression overall followed by the aggregation of unclassified TEs (Fig. 6b). Retroelements are more transcribed than DNA transposons (average $\log _{10} \mathrm{CPM} \sim 2$ and 1.2, respectively).

To understand TE expression dynamics across the pangenome, we investigated associations between TE transcription, length and relative frequency (Fig. 6c). We found TE transcription to be correlated with TE frequency in the genomes (Spearman's $r=0.49, p<5 \mathrm{e}-307$; Fig. 6c) and we found an even stronger correlation at the TE superfamily level (Spearman's $r=0.59, p<5 \mathrm{e}$ $-40)$. However, TE transcription is not correlated with TE length at the superfamily level (Spearman's $r=0.06$, $p>2 \mathrm{e}-1$; Fig. 6c). Interestingly, the average TE transcription levels are positively correlated with TE count in the genome (Fig. 6d). A notable exception is unclassified SINE retroelements. The correlation of TE transcription levels and $\mathrm{TE}$ frequency in the genome strongly suggests that transcriptional activity contributed to recent TE expansions in the genome.

\section{Discussion}

We established a global pangenome of a major fungal wheat pathogen based on the assembly and analysis of 19 high-quality genomes. Z. tritici segregates major chromosomal rearrangements affecting both the more conserved core chromosomes as well as the highly polymorphic accessory chromosomes. The gene content is highly variable among genomes with only $60 \%$ of all genes being conserved in the species. Accessory genes encode functions for a wide variety of interactions with both biotic and abiotic environments. An exhaustive map of TEs across all genomes pinpoints transposon-associated genome expansions across geographic regions.

We showed that the $Z$. tritici pangenome is expansive with $\sim 40 \%$ accessory orthogroups. Compared to a previous construction of the $Z$. tritici pangenome based on genomes from a much narrower geographic breadth [27], we used more relaxed criteria to assign genes into orthogroups. Based on the tendency to assign more divergent gene variants into the same orthogroup, we recovered a total of 911 orthogroups with at least one paralog compared to only 76 identified previously. The number of paralogs remains low compared to species with larger genomes that retained more paralogs of gene duplication events [28]. A likely constraint on gene duplication is the genomic defence mechanism that introduces repeat-induced point (RIP) mutations [46]. Although these defences evolved to suppress transpositional activity of TEs, they can also affect genome evolution by targeting gene duplicates [46, 47]. Recent sequencing efforts oriented around important crop species reported impressively large accessory genome proportions [48-50]. However, nearly all eukaryotic pangenomes are partially based on short-read assemblies that challenge the resolution of segregating gene variants within a species. With the conservative estimate of $24 \%$ non-reference orthogroups, the $Z$. tritici accessory genome is the largest reported for a fungal species to date ( $40 \%$ of the pangenome). This falls outside the upper range of comparative analyses of human fungal pathogens and S. cerevisiae, where estimates of the accessory genome ranged from 10 to $20 \%$ [26]. However, bacterial accessory genomes can range from 0 to $95 \%$ of the total pangenome [3]. The effective population size of a species, its lifestyle, and niche heterogeneity are main factors influencing bacterial pangenome sizes [4]. Similar to bacteria, the effective population size is likely to be the major factor maintaining a large accessory genome in Z. tritici. Previous studies identified Z. tritici as a highly polymorphic species with a rapid decay in linkage disequilibrium, high SNP densities and high recombination rates [32, 35]. As a consequence, the pathogen likely retains significant functional variation within populations as long as the variation is nearly neutral.

Bacterial and fungal genomes show clear functional compartmentalization between core and accessory genes $[4,26]$. In fungi, core orthogroups are enriched for housekeeping functions in contrast to an enrichment for antimicrobial resistance and pathogenicity factors among accessory genes [27]. Here we show that genes encoding carbohydrate-active enzymes (CAZymes) are highly conserved within the species. CAZymes are involved in the degradation of the host cell wall and other storage compounds [51, 52]. Strong conservation of the content in CAZymes may reflect a fundamental adaptation to wheat as a host plant. This contrasts with generalist pathogens, which often evolved larger CAZyme repertoires [53]. In contrast to CAZymes, secondary metabolite gene clusters show substantial presence-absence variation within the species. Fungi produce highly 


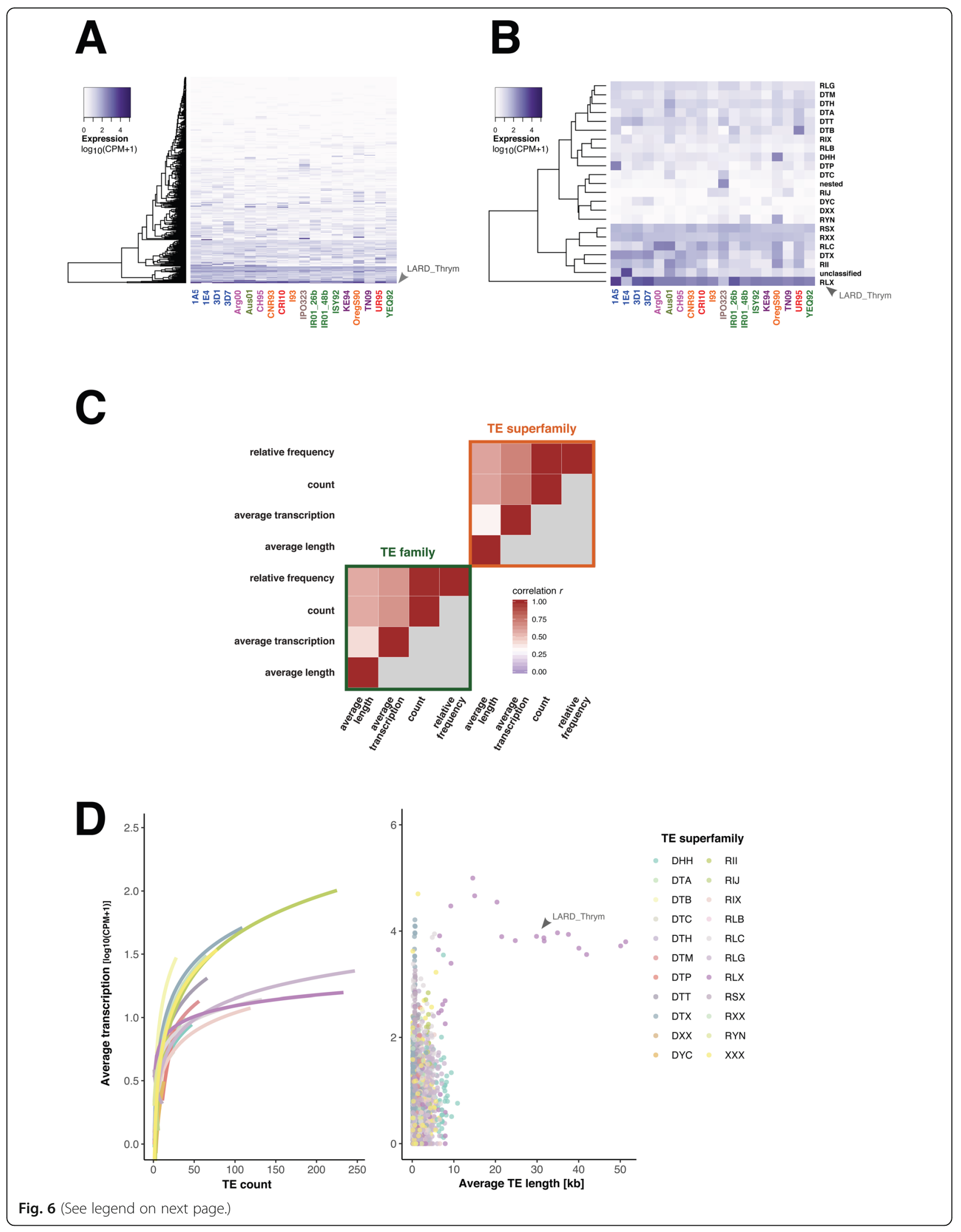


(See figure on previous page.)

Fig. 6 Transcriptional activity of transposable elements (TEs). a TE family transcription levels across all 19 genomes expressed as $\log _{10}(\mathrm{CPM}+1)$. $\mathbf{b}$ Average transcription levels of TE superfamilies across all genomes expressed as average $\log _{10}(C P M+1)$. c Spearman correlation matrix of four TE metrics including counts, relative frequencies, average length and transcription both at the level of TE families and superfamilies. $\mathbf{d}$ Variation of TE transcription (average $\log _{10}(C P M+1)$ ) as a function of TE counts (left panel) or average TE length (right panel). Curves in the left panel show the logarithmic linear regression given by the linear model $\log _{10}(C P M+1) \sim \log _{10}$ (TE count). The highly expressed LARD_Thrym family (RLX) is highlighted using arrows (panels $\mathbf{a}, \mathbf{b}$ and $\mathbf{d}$ )

diverse secondary metabolites that play a role during various life cycle stages, but often have poorly understood functions [54]. Plant pathogens were also shown to depend on secondary metabolite production for full virulence [55]. Hence, variation in secondary metabolite production may underlie variation in virulence. Species from the genus Aspergillus produce a large diversity of secondary metabolites for which the gene clusters often segregate presence-absence $[56,57]$. The $Z$. tritici pangenome was constructed from isolates coming from six different continents and a wide array of agricultural environments. Hence, differences in secondary metabolite production capacity may reflect local adaptation and trade-offs that balance the cost of metabolite production. Virulence of $Z$. tritici is thought to be largely governed by gene-for-gene interactions [58]. In such interactions effector proteins either promote disease or are recognized by the host and trigger resistance [19]. A gene encoding a recognized effector should therefore be rapidly eliminated from the species gene pool. Z. tritici populations responded rapidly to selection on effector gene loci by either mutating, deleting or silencing genes $[21,33,34]$. Our global pangenome analysis significantly expands our understanding of effector gene diversification. We identified 652 orthogroups encoding predicted effector functions of which $63 \%$ are accessory orthogroups. Accessory effector genes may be involved in arms races with strong selection driving the gain or loss of individual effector genes in populations. As a contrast, we identified 45 conserved and highly expressed effectors genes potentially encoding indispensable pathogenicity functions.

Ultimate mechanisms promoting intra-specific diversity in genome structure may include large population sizes and niche complexity, however the proximate mechanisms generating such diversification are poorly understood. TEs can be key drivers generating structural variation $[59,60]$ and $Z$. tritici readily undergoes TEmediated chromosomal rearrangements during meiosis $[38,39]$. Here we show that $Z$. tritici genomes contain $16-24 \%$ TEs, with the overall proportion of TEs accounting for $\sim 70 \%$ of the intraspecific genome size variation. Hence, TEs are key drivers of genome evolution in this species. Among the most drastic chromosomal rearrangements, we detected a significantly shorter chromosome 7 homologue. The longer homologue was hypothesized to have originated from a fusion with an accessory chromosome based on evidence from large-scale epigenetic remodeling [61]. Our analysis likely identified the ancestral variant prior to the suspected chromosomal fusion event. Hence, the species retained two major chromosomal variants of a core chromosome.

TEs are often implicated in gene copy number variation through duplication or pseudogenisation events suggesting that TEs directly contribute to pangenome diversification. We show that specific Gypsy and Helitron elements were integrated into genes generating highly paralogous orthogroups. These orthogroups may underlie recent expansions of specific TEs in the genomes of Australian and Oregon isolates. The Helitron element is among the most transcribed TEs in the Oregon isolate, suggesting a high potential for new transpositions. In contrast, the Gypsy element is only weakly transcribed in the Australian isolate, suggesting that this TE has become deactivated by genomic defences. In addition to transpositional activity causing loss-of-function mutations in genes, TEs can also contribute to genome expansions [62]. We found a strong correlation of TE content and genome size across the pangenome suggesting that TEs are the primary drivers of genome expansions. Because the pathogen was only recently introduced to regions outside of Europe and Asia, genome size variation among geographic regions may have originated from population bottlenecks such as founder events. As an example, populations in Australia underwent a significant founder event during the recent colonization of the continent from Europe [63]. Hence, our observation of an expanded Australian genome may be causally linked to this bottleneck. Genome expansions may also be triggered by TE mobilization. Stressors such as host defences during infection cause substantial TE derepression across the $Z$. tritici genome [45]. Taken together, TE dynamics and large effective population sizes likely constitute the proximate and ultimate drivers of pangenome size evolution. Understanding the birth and death cycles of gene functions in such evolving pangenomes will help address major questions related to croppathogen co-evolution.

\section{Methods}

High molecular-weight DNA extraction and single molecule real-time (SMRT) sequencing

Origin and year of sampling of all the isolates are described in Additional file 2: Table S3. High-molecular- 
weight DNA was extracted from lyophilized spores following a modified version of a cetyltrimethylammonium bromide (CTAB) protocol developed for plant tissue described in [40]. Briefly, $100 \mathrm{mg}$ of lyophilized spores were crushed with a mortar and transferred to a phenolchloroform-isoamyl alcohol solution. The supernatant was centrifuged and the pellet resuspended twice in fresh phenol-chloroform-isoamyl alcohol. The resulting pellet was then washed three times and resuspended in $100 \mu \mathrm{l}$ of sterile water. For each isolate, PacBio SMRTbell libraries were prepared using between $15 \mu \mathrm{g}$ and $30 \mu \mathrm{g}$ of high molecular-weight DNA. Sequencing was performed on a PacBio Sequel instrument at the Functional Genomics Center, Zürich, Switzerland.

\section{Complete genome assemblies}

We largely followed the pipeline described in [64]. In summary, raw PacBio sequencing reads were assembled using Саnu v1.7.1 [65]. All assemblies were performed with an estimated genome size of $39.678 \mathrm{Mb}$ (--genomeSize). Two corrected error rates (--correctedErrorRate 0.045 and 0.039 ) and minimal read length (--minReadLength 500 and 5000) parameters were tested, and the most contiguous chromosome-level assemblies were retained for further analysis based on reference alignment. The scaffolding was quality-controlled by inspecting genome-wide dot plots against previously assembled and validated genomes for reference. For each isolate, raw reads were aligned to the newly assembled genome using pbalign v0.3.1 from Pacific Biosciences suite (https://github.com/PacificBiosciences/pbalign) to inspect potential mis-assemblies. The assemblies were polished twice using PacBio reads mapped back to the new assembly using the software Arrow v2.2.2 from the Pacific Biosciences suite with default settings (https:// github.com/PacificBiosciences/GenomicConsensus) and chromosome-level assemblies were performed using Ragout v2.1.1 and the IPO323 isolate as a reference [66].

\section{RNA extraction, library preparation, sequencing and quantification}

For isolates 1A5, 1E4, 3D1 and 3D7, RNA sequencing experiments on minimal media were performed by [45, 67]. Raw reads were retrieved from the NCBI Short Read Archive accession number SRP077418. Similarly, the 15 additional fungal isolates (Additional file 2: Table S3) were grown in YSB media $(10 \mathrm{~g}$ sucrose $+10 \mathrm{~g}$ yeast extract per litre) and then $10 \mathrm{e} 5$ cells were inoculated on liquid minimal media without a carbon source [68] for 7-10 days prior to extraction to reach identical growth stages as for the previous RNA sequencing experiments. RNA was extracted using a NucleoSpin ${ }^{\circ}$ RNA Plant kit following the manufacturer's instructions. Library preparation was carried out according to the Illumina
TruSeq Stranded mRNA Library Prep protocol with unique indexes for each sample. Single-end 100-bp sequencing was performed on a HiSeq 4000 at the iGE3 platform in Geneva, Switzerland. RNA-seq reads were first filtered using Trimmomatic v0.38 [69] using the following parameters: ILLUMINACLIP:TruSeq3-SE.fa: 2: 30:10 LEADING:10 TRAILING:10 SLIDINGWINDOW: 5:10 MINLEN: 50, and then aligned to the corresponding genome assembly using STAR v2.6.0a [70] allowing for multiple read mapping (parameters set as --outFilterMultimapNmax 100 --winAnchorMultimapNmax 200 --outFilterMismatchNmax 3). We used HTSeq-count v0.11.2 [71] with -s reverse and -m union parameters to recover counts per feature (joint counting of reads in genes and TEs). We calculated normalized feature counts expressed as counts per million, which accounts for library size, using the EdgeR package v3.24.3 [72]. We restricted our analyses to features with a count per million $>1$.

\section{Gene prediction and genome annotation}

We used the gene prediction pipeline BRAKER v2.1 to predict genes in the 14 newly assembled genomes [73-80]. BRAKER combines coding sequence and intron hints based on the mapping of conserved protein sequences and introns identified in RNA-seq data, respectively. The above described RNA-seq datasets were joined with predicted protein sequences from the reference isolate IPO323 [28] and used to predict gene features and guide splice site mapping. RNA alignment files were generated with HISAT2 v2.1.0 using the --rna-strandness $\mathrm{R}$ option [81]. The resulting bam files were provided to BRAKER (--bam option) together with mapped IPO323 reference proteins (--prot_seq option) to generate gene predictions for each assembled genome using the --alternatives-from-evidence $=$ false $\quad-$ prg $=$ gth $\quad$--etpmode --fungus parameters. Orthologous genes were identified using protein sequences from all 19 isolates and Orthofinder v2.1.2 with default parameters [82, 83].

\section{TE consensus identification, classification and annotation}

To obtain consensus sequences for TE families, individual runs of RepeatModeler were performed on the 19 complete genomes in addition to the genome of $Z$. pseudotritici [84]. The classification was based on the GIRI Repbase using RepeatMasker [85, 86]. In order to finalize the classification of TE consensus sequences, we used WICKERsoft [87]. The 19 complete genomes were screened for copies of consensus sequences with blastn filtering for sequence identity of $>80 \%$ on $>80 \%$ of the length of the sequence [88]. Flanks of $300 \mathrm{bp}$ were added and new multiple sequence alignments were performed using ClustalW [89]. Boundaries were visually inspected and trimmed if necessary. Consensus sequences were 
classified according to the presence and type of terminal repeats and homology of encoded proteins using hints from blastx on NCBI. Consensus sequences were renamed according to a three-letter classification system [90].

A second round of annotation was performed based on predicted protein sequences of TE superfamilies from other fungal species. Here again, the 19 complete genomes were screened for a protein sequence of each superfamily using tblastn. Blast hits were filtered for a minimal alignment size of $80 \mathrm{bp}$ and sequence similarity $>35 \%$. Flanks of $3000 \mathrm{bp}$ or more both up- and downstream of the sequence were then added. Hits were pairwise compared with dotplots using dotter and grouped into families based on visual inspection [91]. Finally, multiple sequence alignments were performed with ClustalW to construct consensus sequences and the consensus sequences were renamed according to the three-letter system [90]. TE consensus sequences are available from https://github.com/crolllab/datasets.

A third round of annotation of the 19 complete genomes was done to identify four groups of short nonautonomous TEs. LTR-Finder was used to screen for LARDs (LArge Retrotransposon Derivates) and TRIMs (Terminal Repeat retrotransposons In Miniature) with the filters -d 2001 -D 6000 -1 30 -L 5000 and -d 30 -D 2000 -1 30 -L 500 respectively. MITE-Tracker was used to screen for MITEs (Miniature Inverted-repeat Transposable Elements) and SINE-Finder in Sine-Scan to screen for SINEs (Short Interspersed Nuclear Elements) [92-97]. For each detected LARD, TRIM and SINE, consensus sequences were created as described above and duplicates excluded. All genome assemblies were then annotated with the curated consensus sequences using RepeatMasker with a cut-off value of 250 and ignored simple repeats as well as low complexity regions. Annotated elements shorter than $100 \mathrm{bp}$ were filtered out, and adjacent identical TEs overlapping by more than $100 \mathrm{bp}$ were merged. Different TE families overlapping by more than $100 \mathrm{bp}$ were considered as nested insertions and were renamed accordingly. Identical elements separated by less than $200 \mathrm{bp}$ indicative of putative interrupted elements were grouped into a single element using minimal start and maximal stop positions. TEs overlapping $\geq 1 \mathrm{bp}$ with genes were recovered using the bedtools v2.27.1 suite and the overlap function [98]. Correlations were calculated in RStudio version 1.1.453 using Spearman's coefficient for pairwise complete observations and statistics were inferred with the psych package using the Holm correction method [99].

\section{Functional annotation of predicted genes}

Protein functions were predicted for all gene models using InterProScan v 5.31-70.0 [100] adding -goterms -iprlookup and -pathway information. Secretion peptides and transmembrane domains (TM) were identified using SignalP v 4.1 and Phobius [101, 102]. The secretome was defined as the set of proteins with a signal peptide but no TM as predicted by either SignalP and Phobius. Putative effectors were identified among the set of secreted proteins using EffectorP v 2.0 [103]. Carbohydrate-active enzymes (CAZymes) were identified using dbCAN2 release 7.0 server $[104,105]$ with the three tools HMMER, DIAMOND and Hotpep [106-108]. Proteins were classified as a CAZyme if predicted by each of the three tools. We searched for secondary metabolite gene clusters using the online version 4 of antiSMASH [109]. Genes belonging to an identified cluster were annotated as "biosynthetic", "biosynthetic-additional", "transport", "regulatory" or "other". Gene clusters mapping at a conserved, orthologous locus shared by two or more isolate were considered as syntenic.

\section{Supplementary information}

The online version of this article (https://doi.org/10.1186/s12915-020-0744-3) contains supplementary material, which is available to authorized users.

\footnotetext{
Additional file 1: Figure S1. Integrative Genomics Viewer screenshot of PacBio reads aligned back to the YEQ92 genome assembly at the fusion locus between chromosomes 15 and 16. Figure S2. Percent identity given by the multiple protein sequence alignment for each orthogroup. Protein sequences were aligned using mafft and alignment identity was extracted with the easel alistat tool from Eddy Rivas (https://github.com/ EddyRivasLab/easel). Figure S3. Presence-absence heatmap of the orthogroups assigned to secondary metabolite gene clusters. Each line stands for an orthogroup. Syntenic gene clusters are numbered from 1 to 39. Orthogroups including more than one gene per cluster are shown in darker blue (0-3 genes were found assigned to an orthogroup in this analysis). Figure S4. Evolutionary origins of the secondary metabolite genes clusters. We performed blast searches using all annotated biosynthetic and biosynthetic-additional proteins as query against four closely related sister species of Zymoseptoria tritici. The heatmap shows the percent identity of the top hit found in the four sister species for each of the 147 genes encoding biosynthetic functions in putative gene clusters. The isolates Zpa63, Zp13, Zb87 and Za17 correspond to the species Z. passerinii, Z. pseudotritici, Z. brevis and Z. ardabiliae respectively. Figure S5. Gene expression across pangenome categories. Gene expression is shown as $\log 10$ values of counts per million reads +1 because nonexpressed genes are also shown. Figure S6. Single-gene core orthogroups heatmap following hierarchical clustering based on Euclidian distances. Gene expression is shown as the log10 values of counts per million reads +1 as non-expressed genes are also shown. Figure S7. GC-content across transposable element family consensus sequences. Figure S8. Transposable element (TE) content correlated with genome length for both core and accessory chromosomes. The proportion of TEs was calculated as the percentage of chromosome length in bp. Figure S9. The proportion of the genome covered by genes correlated with total genome size. Figure S10. Heatmap of average transposable element size (log10 of the average length in bp). Figure S11. Heatmap of average transposable element size summarized by superfamily (log10 of the average TE superfamily length in bp). Figure S12. Distance to closest transposable element across pangenome categories given as $\log _{10}$ values of the distance in base pairs. Figure S13. Proportion of pangenome categories overlapping with transposable elements (TE). All features with at least 1 bp overlap with a TE sequence were considered. Figure S14. Proportion of overlapping genes in blue and transposable elements (TE) in grey. All features with at least $1 \mathrm{bp}$ overlap with a TE sequence were considered. Figure S15. Genome-wide transposable element (TE) superfamily frequencies correlated with the proportion of TES overlapping genes. Proportions are given for each TE superfamily (colour
} 
code) and each of the 19 isolates. Figure S16. Frequency of orthogroups showing high ( $>50 \%)$ and low $(<50 \%)$ expression coefficient of variation. Only orthogroups were distinguished whether at least one gene of the orthogroup was located within $100 \mathrm{bp}$ of a transposable element or not. Figure S17. Gene expression as a function of its distance to the closest transposable element (TE). The relationship is shown for each of the TE superfamilies across the 19 isolates. Gene expression is given by the $\log 10$ values of normalized counts per millions reads +1 as genes showing zero expression are also included. Figure $\mathbf{S 1 8}$. Gene expression of genes overlapping at least one base pair with a transposable element ("yes") compared to genes not overlapping ("no"). Gene expression is given by $\log 10$ values of counts per million reads.

Additional file 2: Table S1. List of all identified orthogroups and pangenome categorization. Table S2. List of the genes encoding Major Facilitator Superfamily domains (IPR036259). Table S3. Summary table of the analyzed isolates

\section{Acknowledgements}

We are grateful for helpful comments by Simone Fouché on a previous version of this manuscript. Data generated for this manuscript was obtained in collaboration with the Genetic Diversity Centre (GDC), ETH Zurich and the Functional Genomics Center Zurich (FGCZ).

\section{Authors' contributions}

TB and DC conceived the study; TB and UO performed analyses; LA and BAM provided datasets and strains; BAM and DC provided funding; TB and DC wrote the manuscript. All authors read and approved the final manuscript.

\section{Funding}

BAM and DC received support from the Swiss National Science Foundation (grants 31003A_155955 and 31003A_173265, respectively). DC was also supported by a grant from the Fondation Pierre Mercier pour la Science for this work.

\section{Availability of data and materials}

The genome assembly and annotation for new genome assemblies are available at the European Nucleotide Archive (http://www.ebi.ac.uk/ena) under the BioProject PRJEB33986 [110]. The RNA-sequencing raw sequencing data was deposited at the NCBI Short Read Archive under the accession number PRJNA559981 [111].

\section{Ethics approval and consent to participate}

$\mathrm{N} / \mathrm{a}$

\section{Consent for publication}

N/a

\section{Competing interests}

The authors declare that they have no competing interests.

\section{Author details}

${ }^{1}$ Laboratory of Evolutionary Genetics, Institute of Biology, University of Neuchâtel, Neuchâtel, Switzerland. ${ }^{2}$ Plant Pathology, Institute of Integrative Biology, ETH Zürich, Zürich, Switzerland.

\section{Received: 17 October 2019 Accepted: 27 January 2020}

Published online: 11 February 2020

\section{References}

1. Tettelin H, Riley D, Cattuto C, Medini D. Comparative genomics: the bacterial pan-genome. Curr Opin Microbiol. 2008;11(5):472-7. Available from: http://www.ncbi.nlm.nih.gov/pubmed/19086349. [cited 2019 Jul 19]

2. Ramasamy D, Mishra AK, Lagier J-C, Padhmanabhan R, Rossi M, Sentausa E, et al. A polyphasic strategy incorporating genomic data for the taxonomic description of novel bacterial species. Int J Syst Evol Microbiol. 2014;64(Pt 2): 384-91. Available from: http://www.ncbi.n/m.nih.gov/pubmed/24505076. [cited 2019 Jul 17]

3. Rouli L, Merhej V, Fournier P-E, Raoult D. The bacterial pangenome as a new tool for analysing pathogenic bacteria. New microbes new Infect. 2015;7:
72-85. Available from: http://www.ncbi.nlm.nih.gov/pubmed/26442149. [cited 2019 Jul 17]

4. Mclnerney JO, McNally A, O'Connell MJ. Why prokaryotes have pangenomes. Nat Microbiol. 2017;2(4):17040. Available from: http://www.nature.com/articles/nmicrobiol201740. [cited 2019 Aug 13]

5. Lefébure T, Pavinski Bitar PD, Suzuki H, Stanhope MJ. Evolutionary dynamics of complete campylobacter pan-genomes and the bacterial species concept. Genome Biol Evol. 2010;2:646-55. Available from: http://www.ncbi. nlm.nih.gov/pubmed/20688752. [cited 2019 Jul 17]

6. Sánchez-Vallet A, Fouché S, Fudal I, Hartmann FE, Soyer JL, Tellier A, et al. The genome biology of effector gene evolution in filamentous plant pathogens. Annu Rev Phytopathol. 2018;56(1):21-40. Available from: https://www.annualreviews.org/doi/10.1146/annurev-phyto-080516-035303. [cited 2019 Aug 13]

7. Jackson RW, Vinatzer B, Arnold DL, Dorus S, Murillo J. The influence of the accessory genome on bacterial pathogen evolution. Mob Genet Elements 2011;1(1):55-65. Available from: http://www.ncbi.nlm.nih.gov/pubmed/22 016845. [cited 2019 Aug 26]

8. Wu Y, Zaiden N, Cao B. The core- and pan-genomic analyses of the genus Comamonas: from environmental adaptation to potential virulence. Front Microbiol. 2018;9:3096. Available from: https://www.frontiersin.org/article/1 0.3389/fmicb.2018.03096/full. [cited 2019 Aug 26]

9. Sánchez-Vallet A, Hartmann FE, Marcel TC, Croll D. Nature's genetic screens: using genome-wide association studies for effector discovery. Mol Plant Pathol. 2018;19(1):3-6. Available from: http://www.ncbi.nlm.nih.gov/ pubmed/29226559. [cited 2019 Aug 26]

10. Marschall T, Marz M, Abeel T, Dijkstra L, Dutilh BE, Ghaffaari A, et al. Computational pan-genomics: status, promises and challenges. Brief Bioinform. 2016;19(1):bbw089. Available from: https://academic.oup.com/ bib/article-lookup/doi/10.1093/bib/bbw089. [cited 2019 Aug 26]

11. Schrider DR, Hahn MW. Gene copy-number polymorphism in nature. Proc R Soc B Biol Sci. 2010;277(1698):3213-21. Available from: http://www.ncbi.n/m. nih.gov/pubmed/20591863. [cited 2019 Aug 13]

12. Brynildsrud O, Gulla S, Feil EJ, Nørstebø SF, Rhodes LD. Identifying copy number variation of the dominant virulence factors msa and p22 within genomes of the fish pathogen Renibacterium salmoninarum. Microb genomics. 2016;2(4):e000055. Available from: http://www.ncbi.nlm.nih.gov/ pubmed/28348850. [cited 2019 Jul 19]

13. Plissonneau C, Daverdin G, Ollivier B, Blaise F, Degrave A, Fudal I, et al. A game of hide and seek between avirulence genes AvrLm4-7 and AvrLm3 in Leptosphaeria maculans. New Phytol. 2016;209(4):1613-24. Available from: http://www.ncbi.nlm.nih.gov/pubmed/26592855. [cited 2017 Sep 13]

14. Hartmann FE, Rodríguez de la Vega RC, Brandenburg J-T, Carpentier F, Giraud T. Gene presence-absence polymorphism in castrating Anther-Smut fungi: recent gene gains and phylogeographic structure. Van De Peer $Y$, editor. Genome Biol Evol. 2018;10(5):1298-314. Available from: https:// academic.oup.com/gbe/article/10/5/1298/4990910. [cited 2019 Jul 19]

15. Araki H, Tian D, Goss EM, Jakob K, Halldorsdottir SS, Kreitman M, et al. Presence/absence polymorphism for alternative pathogenicity islands in Pseudomonas viridiflava, a pathogen of Arabidopsis. Pnas. 2006;103(15): 5887-92.

16. De PJGM W, Mehrabi R, Van den HA B, Stergiopoulos I. Fungal effector proteins: past, present and future. Mol Plant Pathol. 2009:10(6):735-47. Available from: http://www.ncbi.nlm.nih.gov/pubmed/19849781. [cited 2017 Sep 18]

17. Lo Presti L, Lanver D, Schweizer G, Tanaka S, Liang L, Tollot M, et al. Fungal effectors and plant susceptibility. Annu Rev Plant Biol. 2015;66(1):513-45. Available from: http://www.ncbi.nlm.nih.gov/pubmed/25923844. [cited 2017 Sep 13]

18. Toruño TY, Stergiopoulos I, Coaker G. Plant-pathogen effectors: cellular probes interfering with plant defenses in spatial and temporal manners. Annu Rev Phytopathol. 2016;54(1):419-41. Available from: http://www.annualreviews.org/ doi/10.1146/annurev-phyto-080615-100204. [cited 2019 Aug 26]

19. Jones JDG, Dangl JL. The plant immune system. Nature. 2006:444(7117): 323-9. Available from: http://www.ncbi.nlm.nih.gov/pubmed/17108957. [cited 2014 Jul 10]

20. Yoshida K, Saunders DGO, Mitsuoka C, Natsume S, Kosugi S, Saitoh H, et al Host specialization of the blast fungus Magnaporthe oryzae is associated with dynamic gain and loss of genes linked to transposable elements. BMC Genomics. 2016;17(1):370. Available from: http://bmcgenomics. biomedcentral.com/articles/10.1186/s12864-016-2690-6. [cited 2019 Jul 19]

21. Hartmann FE, Croll D. Distinct trajectories of massive recent gene gains and losses in populations of a microbial eukaryotic pathogen. Mol Biol Evol. 
2017;127(19):1-18. Available from: https://academic.oup.com/mbe/articlelookup/doi/10.1093/molbev/msx208. [cited 2017 Sep 13]

22. Faino L, Seidl MF, Shi-Kunne X, Pauper M, Van Den Berg GCM, Wittenberg AHJ, et al. Transposons passively and actively contribute to evolution of the twospeed genome of a fungal pathogen. Genome Res. 2016;26(8):1091-100.

23. Sperschneider J, Gardiner DM, Thatcher LF, Lyons R, Singh KB, Manners JM, et al. Genome-wide analysis in three Fusarium pathogens identifies rapidly evolving chromosomes and genes associated with pathogenicity. Genome Biol Evol. 2015;7(6):1613-27. Available from: http://www.ncbi.nlm.nih.gov/ pubmed/25994930. [cited 2017 Sep 13]

24. Dong S, Raffaele S, Kamoun S. The two-speed genomes of filamentous pathogens: waltz with plants. Curr Opin Genet Dev. 2015;35:57-65. Available from: http://www.ncbi.nlm.nih.gov/pubmed/26451981. [cited 2019 Jul 19]

25. Fouché S, Plissonneau C, Croll D. The birth and death of effectors in rapidly evolving filamentous pathogen genomes. Curr Opin Microbiol. 2018:46:3442. Available from: http://www.ncbi.nlm.nih.gov/pubmed/29455143. [cited 2019 Aug 13]

26. McCarthy CGP, Fitzpatrick DA. Pan-genome analyses of model fungal species. Microb genomics. 2019;5(2) Available from: http://www.ncbi.nlm. nih.gov/pubmed/30714895. [cited 2019 Jul 19]

27. Plissonneau C, Hartmann FE, Croll D. Pangenome analyses of the wheat pathogen Zymoseptoria tritici reveal the structural basis of a highly plastic eukaryotic genome. BMC Biol. 2018;16(1):5. Available from: https://bmcbiol. biomedcentral.com/articles/10.1186/s12915-017-0457-4. [cited 2018 Feb 6]

28. Goodwin SB, Ben M'Barek S, Dhillon B, Wittenberg AHJ, Crane CF, Hane JK, et al. Finished genome of the fungal wheat pathogen Mycosphaerella graminicola reveals dispensome structure, chromosome plasticity, and stealth pathogenesis. Malik HS, editor. PLoS Genet. 2011;7(6):e1002070. Available from: https://dx.plos.org/10.1371/journal.pgen.1002070. [cited 2019 Jun 6]

29. Cools HJ, Fraaije BA. Are azole fungicides losing ground against Septoria wheat disease? Resistance mechanisms inMycosphaerella graminicola. Pest Manag Sci. 2008;64(7):681-4. Available from: http://doi.wiley.com/10.1002/ ps.1568. [cited 2019 Aug 13]

30. Blake JJ, Gosling P, Fraaije BA, Burnett FJ, Knight SM, Kildea S, et al. Changes in field dose-response curves for demethylation inhibitor (DMI) and quinone outside inhibitor (Qol) fungicides against Zymoseptoria tritici related to laboratory sensitivity phenotyping and genotyping assays. Pest Manag Sci. 2018;74(2):302-13. Available from: http://doi.wiley.com/10.1002/ ps.4725. [cited 2019 Aug 13]

31. Lucas JA, Hawkins NJ, Fraaije BA. The evolution of fungicide resistance. In: Advances in applied microbiology; 2015. p. 29-92. Available from: http://www.ncbi.nlm.nih.gov/pubmed/25596029. [cited 2019 Aug 13].

32. Hartmann FE, Sánchez-Vallet A, McDonald BA, Croll D. A fungal wheat pathogen evolved host specialization by extensive chromosomal rearrangements. ISME J. 2017;11(5):1189-204. Available from: http://www. ncbi.nlm.nih.gov/pubmed/28117833. [cited 2017 Sep 13]

33. Meile L, Croll D, Brunner PC, Plissonneau C, Hartmann FE, McDonald BA, et al. A fungal avirulence factor encoded in a highly plastic genomic region triggers partial resistance to septoria tritici blotch. New Phytol. 2018;219(3): 1048-61. Available from: http://www.ncbi.nlm.nih.gov/pubmed/29693722. [cited 2019 Aug 26]

34. Krishnan P, Meile L, Plissonneau C, Ma X, Hartmann FE, Croll D, et al. Transposable element insertions shape gene regulation and melanin production in a fungal pathogen of wheat. BMC Biol. 2018;16(1):78. Available from: http://www.ncbi.nlm.nih.gov/pubmed/30012138. [cited 2019 Aug 26]

35. Croll D, Lendenmann MH, Stewart E, BA MD. The impact of recombination hotspots on genome evolution of a fungal plant pathogen. Genetics. 2015; 201(3):1213-28. Available from: http://www.ncbi.nlm.nih.gov/pubmed/263 92286. [cited 2017 Aug 19]

36. Grandaubert J, Dutheil JY, Stukenbrock EH. The genomic determinants of adaptive evolution in a fungal pathogen. Evol Lett. 2019;3(3):299-312. Available from: http://doi.wiley.com/10.1002/evl3.117. [cited 2019 Aug 26]

37. Stukenbrock EH, Dutheil JY. Fine-scale recombination maps of fungal plant pathogens reveal dynamic recombination landscapes and intragenic hotspots. Genetics. 2018;208(3):1209-29. Available from: http://www.ncbi. nlm.nih.gov/pubmed/29263029. [cited 2019 Aug 26]

38. Croll D, Zala M, McDonald BA, Smoot M, Shumway M. Breakage-fusionbridge cycles and large insertions contribute to the rapid evolution of accessory chromosomes in a fungal pathogen. Heitman J, editor. PLoS
Genet. 2013;9(6):e1003567. Available from: http://dx.plos.org/10.1371/journal. pgen.1003567. [cited 2017 Sep 13]

39. Fouché S, Plissonneau C, McDonald BA, Croll D. Meiosis leads to pervasive copy-number variation and distorted inheritance of accessory chromosomes of the wheat pathogen Zymoseptoria tritici. Genome Biol Evol. 2018;10(6):1416-29. Available from: http://www.ncbi.nlm.nih.gov/ pubmed/29850789. [cited 2019 Aug 26]

40. Plissonneau C, Stürchler A, Croll D. The evolution of orphan regions in genomes of a fungal pathogen of wheat. MBio. 2016;7((5):e01231-16. Available from: http://www.ncbi.nlm.nih.gov/pubmed/27795389. [cited 2019 Feb 22]

41. Steinberg G. Cell biology of Zymoseptoria tritici: pathogen cell organization and wheat infection. Fungal Genet Biol. 2015;79:17-23. Available from: http://www.ncbi.nlm.nih.gov/pubmed/26092785. [cited 2019 Aug 13]

42. Palma-Guerrero J, Ma X, Torriani SFF, Zala M, Francisco CS, Hartmann FE, et al. Comparative transcriptome analyses in Zymoseptoria tritici reveal significant differences in gene expression among strains during plant infection. Mol Plant-Microbe Interact. 2017:30(3):231-44. Available from: http://www.ncbi.nlm.nih.gov/pubmed/28121239. [cited 2017 Sep 13]

43. Steinhauer D, Salat M, Frey R, Mosbach A, Luksch T, Balmer D, et al. A dispensable paralog of succinate dehydrogenase subunit $C$ mediates standing resistance towards a subclass of SDHI fungicides in Zymoseptoria tritici. bioRxiv. 2019:616904. Available from: https:/www.biorxiv.org/ content/10.1101/616904v1. [cited 2019 Jul 8]

44. Omrane S, Audéon C, Ignace A, Duplaix C, Aouini L, Kema G, et al. Plasticity of the MFS1 promoter leads to multidrug resistance in the wheat pathogen Zymoseptoria tritici. mSphere. 2017;2(5):e00393-17. Available from: http:// www.ncbi.nlm.nih.gov/pubmed/29085913. [cited 2019 Sep 12]

45. Fouché S, Badet T, Oggenfuss U, Plissonneau C, Francisco CS, Croll D. Stressdriven transposable element de-repression dynamics and virulence evolution in a fungal pathogen. Arkhipova I, editor. Mol Biol Evol. 2019; Available from: https:/academic.oup.com/mbe/advance-article/doi/10.1093/ molbev/msz216/5573762. [cited 2019 Oct 2]

46. Selker EU. Repeat-induced gene silencing in fungi. Adv Genet. 2002;46:43950. Available from: https:/www.sciencedirect.com/science/article/abs/pii/ S0065266002460166?via\%3Dihub. [cited 2019 Aug 26]

47. Galagan JE, Selker EU. RIP: the evolutionary cost of genome defense. Trends Genet. 2004;20(9):417-23. Available from: http://www.ncbi.nIm.nih.gov/ pubmed/15313550. [cited 2019 Aug 26]

48. Hirsch CN, Foerster JM, Johnson JM, Sekhon RS, Muttoni G, Vaillancourt B, et al. Insights into the maize pan-genome and pan-transcriptome. Plant Cell. 2014;26(1):121-35. Available from: http://www.ncbi.nlm.nih.gov/ pubmed/24488960. [cited 2019 Aug 16]

49. Zhou P, Silverstein KAT, Ramaraj T, Guhlin J, Denny R, Liu J, et al. Exploring structural variation and gene family architecture with De Novo assemblies of 15 Medicago genomes. BMC Genomics. 2017;18(1):261. Available from: http://www.ncbi.nlm.nih.gov/pubmed/28347275. [cited 2019 Aug 16]

50. Zhao Q, Feng Q, Lu H, Li Y, Wang A, Tian Q, et al. Pan-genome analysis highlights the extent of genomic variation in cultivated and wild rice. Nat Genet. 2018;50(2):278-84. Available from: http://www.nature.com/articles/ s41588-018-0041-z. [cited 2019 Jul 19]

51. Lyu X, Shen C, Fu Y, Xie J, Jiang D, Li G, et al. Comparative genomic and transcriptional analyses of the carbohydrate-active enzymes and secretomes of phytopathogenic fungi reveal their significant roles during infection and development. Sci Rep. 2015;5(1):15565. Available from: http://www.nature. com/articles/srep15565. [cited 2019 Aug 16]

52. Zerillo MM, Adhikari BN, Hamilton JP, Buell CR, Lévesque CA, Tisserat N. Carbohydrate-active enzymes in pythium and their role in plant cell wall and storage polysaccharide degradation. Lespinet O, editor. PLoS One. 2013;8(9): e72572. Available from: https://dx.plos.org/10.1371/journal.pone.0072572. [cited 2019 Aug 16]

53. Zhao Z, Liu H, Wang C, Xu J-R. Comparative analysis of fungal genomes reveals different plant cell wall degrading capacity in fungi. BMC Genomics. 2013;14(1):274. Available from: http:/www.ncbi.nlm.nih.gov/pubmed/2361 7724. [cited 2017 Sep 18]

54. Calvo AM, Wilson RA, Bok JW, Keller NP. Relationship between secondary metabolism and fungal development. Microbiol Mol Biol Rev. 2002;66(3): 447-59. table of contents. Available from: http://www.ncbi.nlm.nih.gov/ pubmed/12208999. [cited 2019 Aug 16]

55. Pusztahelyi T, Holb IJ, Pócsi I. Secondary metabolites in fungus-plant interactions. Front Plant Sci. 2015;6:573. Available from: http://www.ncbi.nlm. nih.gov/pubmed/26300892. [cited 2019 Aug 16] 
56. Raffa N, Keller NP. A call to arms: mustering secondary metabolites for success and survival of an opportunistic pathogen. Sheppard DC, editor. PLOS Pathog. 2019;15(4):e1007606. Available from: http://dx.plos.org/10.13 71/journal.ppat.1007606. [cited 2019 Aug 26]

57. Kjærbølling I, Vesth TC, Frisvad JC, Nybo JL, Theobald S, Kuo A, et al. Linking secondary metabolites to gene clusters through genome sequencing of six diverse Aspergillus species. Proc Natl Acad Sci U S A. 2018;115(4):E753-61. Available from: http//www.ncbi.nlm.nih.gov/pubmed/29317534. [cited 2019 Aug 26]

58. Brown JKM, Chartrain L, Lasserre-Zuber P, Saintenac C. Genetics of resistance to Zymoseptoria tritici and applications to wheat breeding. Fungal Genet Biol. 2015;79:33-41. Available from: http://www.ncbi.nlm.nih. gov/pubmed/26092788. [cited 2019 Aug 26]

59. Beck CR, Garcia-Perez JL, Badge RM, Moran JV. LINE-1 elements in structural variation and disease. Annu Rev Genomics Hum Genet. 2011;12(1):187-215. Available from: http://www.annualreviews.org/doi/10.1146/annurev-genom082509-141802. [cited 2019 Feb 20]

60. Kim S, Mun S, Kim T, Lee K-H, Kang K, Cho J-Y, et al. Transposable elementmediated structural variation analysis in dog breeds using whole-genome sequencing. Mamm Genome. 2019; Available from: http://www.ncbi.nlm.nih. gov/pubmed/31414176. [cited 2019 Aug 26]

61. Schotanus K, Soyer JL, Connolly LR, Grandaubert J, Happel P, Smith KM, et al. Histone modifications rather than the novel regional centromeres of Zymoseptoria tritici distinguish core and accessory chromosomes. Epigenetics Chromatin. 2015;8(1):41. Available from: http://www. epigeneticsandchromatin.com/content/8/1/41. [cited 2019 Jul 8]

62. Naville M, Henriet S, Warren I, Sumic S, Reeve M, Volff J-N, et al. Massive changes of genome size driven by expansions of non-autonomous transposable elements. Curr Biol. 2019;29(7):1161-1168.e6. Available from: https://www.sciencedirect.com/science/article/abs/pii/S0960982219301393. [cited 2019 Aug 19]

63. Zhan J, Linde CC, Jurgens T, Merz U, Steinebrunner F, BA MD. Variation for neutral markers is correlated with variation for quantitative traits in the plant pathogenic fungus Mycosphaerella graminicola. Mol Ecol. 2005;14(9): 2683-93. Available from: http://doi.wiley.com/10.1111/j.1365-294X.2005.0263 8.x. [cited 2019 Sep 12]

64. Yue J-X, Liti G. Long-read sequencing data analysis for yeasts. Nat Protoc. 2018;13(6):1213-31. Available from: http://www.nature.com/articles/nprot.2 018.025. [cited 2019 Jul 16]

65. Koren S, Walenz BP, Berlin K, Miller JR, Bergman NH, Phillippy AM. Canu: scalable and accurate long-read assembly via adaptive k-mer weighting and repeat separation. Genome Res. 2017;27(5):722-36. Available from: http://www.ncbi.nlm.nih.gov/pubmed/28298431. [cited 2019 Jul 16]

66. Kolmogorov M, Raney B, Paten B, Pham S. Ragout-a reference-assisted assembly tool for bacterial genomes. Bioinformatics. 2014;30(12):i302-9 Available from: http://www.ncbi.nlm.nih.gov/pubmed/24931998. [cited 2019 Jul 16]

67. Francisco CS, Ma X, Zwyssig MM, McDonald BA, Palma-Guerrero J. Morphological changes in response to environmental stresses in the fungal plant pathogen Zymoseptoria tritici. Sci Rep. 2019;9(1):9642. Available from: http://www.nature.com/articles/s41598-019-45994-3. [cited 2019 Oct 2]

68. Metzenberg RL. Vogel's Medium N salts: avoiding the need for ammonium nitrate. Fungal Genet Rep. 2003;50(1):14. Available from: https://newprairiepress.org/fgr/vol50/iss1/6. [cited 2019 Jul 16]

69. Bolger AM, Lohse M, Usadel B. Trimmomatic: a flexible trimmer for Illumina sequence data. Bioinformatics. 2014;30(15):2114-20. Available from: http://www.ncbi.nlm.nih.gov/pubmed/24695404. [cited 2019 Jul 16]

70. Dobin A, Davis CA, Schlesinger F, Drenkow J, Zaleski C, Jha S, et al. STAR: ultrafast universal RNA-seq aligner. Bioinformatics. 2013;29(1):15-21. Available from: http://www.ncbi.nlm.nih.gov/pubmed/23104886. [cited 2019 Aug 16]

71. Anders S, Pyl PT, Huber W. HTSeq--a Python framework to work with highthroughput sequencing data. Bioinformatics. 2015;31(2):166-9. Available from: http://www.ncbi.nlm.nih.gov/pubmed/25260700. [cited 2019 Jul 16]

72. Robinson MD, McCarthy DJ, Smyth GK. edgeR: a Bioconductor package for differential expression analysis of digital gene expression data. Bioinformatics. 2010;26(1):139. Available from: http://www.ncbi.nlm.nih.gov/ pubmed/19910308. [cited 2019 Jul 16];

73. Hoff KJ, Lange S, Lomsadze A, Borodovsky M, Stanke M. BRAKER1: Unsupervised RNA-Seq-Based Genome Annotation with GeneMark-ET and AUGUSTUS: Table 1. Bioinformatics. 2016;32(5):767-9. Available from: http://www.ncbi.nlm.nih.gov/pubmed/26559507. [cited 2019 Jul 16]
74. Stanke M, Schöffmann O, Morgenstern B, Waack S. Gene prediction in eukaryotes with a generalized hidden Markov model that uses hints from external sources. BMC Bioinformatics. 2006;7:62. Available from: http://www. ncbi.nlm.nih.gov/pubmed/16469098. [cited 2019 Jul 16]

75. Stanke M, Diekhans M, Baertsch R, Haussler D. Using native and syntenically mapped CDNA alignments to improve de novo gene finding. Bioinformatics. 2008;24(5):637-44. Available from: http://www.ncbi.nlm.nih. gov/pubmed/18218656. [cited 2019 Jul 16]

76. Altschul SF, Gish W, Miller W, Myers EW, Lipman DJ. Basic local alignment search tool. J Mol Biol. 1990;215(3):403-10. Available from: http://www.ncbi. nlm.nih.gov/pubmed/2231712. [cited 2014 Jul 10]

77. Camacho C, Coulouris G, Avagyan V, Ma N, Papadopoulos J, Bealer K, et al. BLAST+ architecture and applications. BMC Bioinformatics. 2009;10(1):421. Available from: http://unw.biomedcentral.com/1471-2105/10/421. [cited 2019 Jul 16]

78. Lomsadze A, Burns PD, Borodovsky M. Integration of mapped RNA-Seq reads into automatic training of eukaryotic gene finding algorithm. Nucleic Acids Res. 2014;42(15):e119. Available from: http://www.ncbi.nlm.nih.gov/ pubmed/24990371. [cited 2019 Jul 16]

79. Barnett DW, Garrison EK, Quinlan AR, Stromberg MP, Marth GT. BamTools: a $\mathrm{C}++\mathrm{API}$ and toolkit for analyzing and managing BAM files. Bioinformatics. 2011;27(12):1691-2. Available from: https://academic.oup.com/bioinformatics/ article-lookup/doi/10.1093/bioinformatics/btr174. [cited 2019 Jul 16]

80. Li H, Handsaker B, Wysoker A, Fennell T, Ruan J, Homer N, et al. The Sequence Alignment/Map format and SAMtools. Bioinformatics. 2009;25(16): 2078-9. Available from: https://academic.oup.com/bioinformatics/articlelookup/doi/10.1093/bioinformatics/btp352. [cited 2019 Jul 16]

81. Kim D, Langmead B, Salzberg SL. HISAT: a fast spliced aligner with low memory requirements. Nat Methods. 2015;12(4):357-60. Available from: http://www.nature.com/articles/nmeth.3317. [cited 2019 Jul 16]

82. Emms DM, Kelly S. OrthoFinder: solving fundamental biases in whole genome comparisons dramatically improves orthogroup inference accuracy. Genome Biol. 2015;16(1):157. Available from: http://genomebiology.com/2 015/16/1/157. [cited 2019 Jul 16]

83. Emms DM, Kelly S. OrthoFinder: phylogenetic orthology inference for comparative genomics. bioRxiv. 2019:466201. Available from: https://www. biorxiv.org/content/10.1101/466201v2.full. [cited 2019 Jul 16]

84. Stukenbrock EH, Jørgensen FG, Zala M, Hansen TT, BA MD, Schierup MH. Whole-genome and chromosome evolution associated with host adaptation and speciation of the wheat pathogen Mycosphaerella graminicola. Malik HS, editor. PLoS Genet. 2010;6(12):e1001189. Available from: http://dx.plos.org/10.1371/journal.pgen.1001189. [cited 2018 Aug 20]

85. Smit, AFA, Hubley, R \& Green P. RepeatMasker Open-4.0 2015. Available from: http://repeatmasker.org

86. Bao W, Kojima KK, Kohany O. Repbase Update, a database of repetitive elements in eukaryotic genomes. Mob DNA. 2015;6(1):11. Available from: http://www.mobilednajournal.com/content/6/1/11. [cited 2019 Jul 16]

87. Breen J, Wicker T, Kong X, Zhang J, Ma W, Paux E, et al. A highly conserved gene island of three genes on chromosome $3 B$ of hexaploid wheat: diverse gene function and genomic structure maintained in a tightly linked block. BMC Plant Biol. 2010;10:98. Available from: http://www.ncbi.nlm.nih.gov/ pubmed/20507561. [cited 2019 Jul 16]

88. Altschul SF, Madden TL, Schäffer AA, Zhang J, Zhang Z, Miller W, et al. Gapped BLAST and PSI-BLAST: a new generation of protein database search programs. Nucleic Acids Res. 1997;25(17):3389-402.

89. Higgins DG, Sharp PM. CLUSTAL: a package for performing multiple sequence alignment on a microcomputer. Gene. 1988;73(1):237-44.

90. Wicker T, Sabot F, Hua-Van A, Bennetzen JL, Capy P, Chalhoub B, et al. A unified classification system for eukaryotic transposable elements. Nat Rev Genet. 2007;8(12):973-82.

91. Sonnhammer ELL, Durbin R. A dot-matrix program with dynamic threshold control suited for genomic DNA and protein sequence analysis. Gene. 1995; 167(1-2):GC1-10.

92. Xu Z, Wang H. LTR-FINDER: an efficient tool for the prediction of full-length LTR retrotransposons. Nucleic Acids Res. 2007;35(SUPPL.2):265-8.

93. Gao D, Li Y, Do KK, Abernathy B, Jackson SA. Landscape and evolutionary dynamics of terminal repeat retrotransposons in miniature in plant genomes. Genome Biol. 2016;17(1):7. Available from: http://www.ncbi.nlm. nih.gov/pubmed/26781660. [cited 2019 Jul 16]

94. Ma B, Li T, Xiang Z, He N. MnTEdb, a collective resource for mulberry transposable elements. Database. 2015;2015 Available from: https:/academic.oup. com/database/article/doi/10.1093/database/bav004/2433136 [cited 2019 Jul 16] 
95. Crescente JM, Zavallo D, Helguera M, Vanzetti LS. MITE Tracker: an accurate approach to identify miniature inverted-repeat transposable elements in large genomes. BMC Bioinformatics. 2018;19(1):348. Available from: https://bmcbioinformatics.biomedcentral.com/articles/10.1186/s12859-01 8-2376-y. [cited 2019 Jul 16]

96. Mao H, Wang H. SINE scan: an efficient tool to discover short interspersed nuclear elements (SINEs) in large-scale genomic datasets. Bioinformatics. 2017;33(5):btw718. Available from: http://www.ncbi.nlm.nih.gov/pubmed/2 8062442. [cited 2019 Jul 16]

97. Wenke T, Dobel T, Sorensen TR, Junghans H, Weisshaar B, Schmidt T. Targeted identification of short interspersed nuclear element families shows their widespread existence and extreme heterogeneity in plant genomes. Plant Cell Online. 2011;23(9):3117-28.

98. Quinlan AR, Hall IM. BEDTools: a flexible suite of utilities for comparing genomic features. Bioinformatics. 2010;26(6):841-2. Available from: https://academic.oup.com/bioinformatics/article-lookup/doi/10.1093/ bioinformatics/btq033. [cited 2019 Jul 16]

99. Revelle WR. psych: Procedures for Personality and Psychological Research. 2017 Available from: https://www.scholars.northwestern.edu/en/ publications/psych-procedures-for-personality-and-psychological-research. [cited 2019 Aug 16]

100. Jones P, Binns D, Chang H-Y, Fraser M, Li W, McAnulla C, et al. InterProScan 5 : genome-scale protein function classification. Bioinformatics. 2014;30(9):123640. Available from: http://www.pubmedcentral.nih.gov/articlerender.fcgi?artid= 3998142\&tool=pmcentrez\&rendertype=abstract. [cited 2014 Jul 13]

101. Petersen TN, Brunak S, von Heijne G, Nielsen H. SignalP 4.0: discriminating signal peptides from transmembrane regions. Nat Methods. 2011;8(10):7856. Available from: http://www.ncbi.n/m.nih.gov/pubmed/21959131. [cited 2019 Jul 16]

102. Käll L, Krogh A, Sonnhammer EL. A combined transmembrane topology and signal peptide prediction method. J Mol Biol. 2004;338(5):1027-36. Available from: http://www.ncbi.nlm.nih.gov/pubmed/15111065. [cited 2019 Jul 16]

103. Sperschneider J, Gardiner DM, Dodds PN, Tini F, Covarelli L, Singh KB, et al. Effector P: predicting fungal effector proteins from secretomes using machine learning. New Phytol. 2016;210(2):743-61. Available from: http://www.ncbi.nlm.nih.gov/pubmed/26680733. [cited 2019 Jul 16]

104. Zhang H, Yohe T, Huang L, Entwistle S, Wu P, Yang Z, et al. dbCAN2: a meta server for automated carbohydrate-active enzyme annotation. Nucleic Acids Res. 2018;46(W1):W95-101. Available from: http://www.ncbi.n/m.nih.gov/ pubmed/29771380. [cited 2019 Jul 16]

105. Lombard V, Golaconda Ramulu H, Drula E, Coutinho PM, Henrissat B. The carbohydrate-active enzymes database (CAZy) in 2013. Nucleic Acids Res. 2014;42(Database issue):D490-5. Available from: https://academic.oup.com/ nar/article-lookup/doi/10.1093/nar/gkt1178. [cited 2019 Jul 16]

106. Finn RD, Clements J, Eddy SR. HMMER web server: interactive sequence similarity searching. Nucleic Acids Res. 2011;39(web server issue):W29-37. Available from: http://www.ncbi.n/m.nih.gov/pubmed/21593126. [cited 2019 Jul 16]

107. Buchfink $B$, Xie C, Huson DH. Fast and sensitive protein alignment using DIAMOND. Nat Methods. 2015;12(1):59-60. Available from: http://www.ncbi. nlm.nih.gov/pubmed/25402007. [cited 2019 Jul 16]

108. Busk PK, Pilgaard B, Lezyk MJ, Meyer AS, Lange L. Homology to peptide pattern for annotation of carbohydrate-active enzymes and prediction of function. BMC Bioinformatics. 2017;18(1):214. Available from: http://www. ncbi.nlm.nih.gov/pubmed/28403817. [cited 2019 Jul 16]

109. Blin K, Wolf T, Chevrette MG, Lu X, Schwalen CJ, Kautsar SA, et al. antiSMASH 4.0-improvements in chemistry prediction and gene cluster boundary identification. Nucleic Acids Res. 2017:45(W1):W36-41. Available from: http://www.ncbi.nlm.nih.gov/pubmed/28460038. [cited 2019 Jul 16]

110. Badet T, Oggenfuss U, Abraham L, McDonald BA, Croll D. A 19-isolate reference-quality global pangenome for the fungal wheat pathogen Zymoseptoria tritici. Supplementary Datasets. 2020. ENA accession: PRJEB33986 https://www.ebi.ac.uk/ena/data/search?query=PRJEB33986

111. Badet T, Oggenfuss U, Abraham L, McDonald BA, Croll D. A 19-isolate reference-quality global pangenome for the fungal wheat pathogen Zymoseptoria tritici. Supplementary Datasets. 2020. NCBI accession: PRJNA559981 https://www.ncbi.nlm.nih.gov/bioproject/PRJNA559981

\section{Publisher's Note}

Springer Nature remains neutral with regard to jurisdictional claims in published maps and institutional affiliations.

\section{Ready to submit your research? Choose BMC and benefit from:}

- fast, convenient online submission

- thorough peer review by experienced researchers in your field

- rapid publication on acceptance

- support for research data, including large and complex data types

- gold Open Access which fosters wider collaboration and increased citations

- maximum visibility for your research: over $100 \mathrm{M}$ website views per year

At $\mathrm{BMC}$, research is always in progress.

Learn more biomedcentral.com/submissions 